(Aus der König1. Universitäts-Frauenklinik in Leipzig.)

\title{
Zur Prognose der ascendirten Gonorrhoe beim Weibe.
}

Von

Dr. B. Kroenig, a. o. Professor in Leipzig.

Bei der Besprechung der entzündlichen Erkrankung der Tuben und des Beckenbanchfelles im "Handbuch der Gynäkologie" sagt Veit: "Den übermässigen Operationen folgen immer Zeiten von Sammlung und Versuchen, conservativ vorzugehen". Bei der ascendirten Gonorrhoe, bei den gonorrhoischen Adnexerkrankungen befindet sich die Gynäkologie in Deutschland z. Zt. entschieden im letzteren Stadium.

Wenn an Stelle der operativen Hochfluth heute mehr eine conservative Methode getreten ist, so kann dies seinen Grund darin haben, dass entweder die operativen Maassnahmen nicht den erwarteten Erfolg gehabt haben, oder auch darin, dass man den klinischen Ablauf der gonorrhoischen Adnexerkrankungen zu ungünstig beurtheilt hat, sodass man eine schwere, oft lebensgefährliche Operation nicht mehr einsetzen will, wenn ein exspectatives Verfahren in nicht allzu langer Zeit ebenfalls zur Heilung oder wenigstens zur Besserung der Beschwerden führen kann.

Im Anfang der 90er Jahre hat die Leipziger Klinik unter dem Eindruck der damaligen Ansichten über die pathologische Dignität der gonorrhoisehen Adnexerkrankungen ebenfalls eine grosse Zahl von Operationen wegen dieser Erkrankungen ausgeführt, wenn eine längere Beobachtungszeit nicht bald Besserung der Beschwerden der Patientinnen brachte.

Die operative Behandlung bestand anfänglich darin, dass die- 
392 Kroenig, Zur Prognose der ascendirten Gonorrhoe beim Weibe.

jenigen Organe, an welchen makroskopisch am deutlichsten Veränderungen nachgewiesen werden konnten, entfernt wurden; so wurden zunächst ausschliesslich die mit Eiter gefüllten Tubensäcke exstirpirt, dagegen wurden der Uterus und die Ovarien gewöhnlich zurückgelassen. Der primäre Erfolg der Operation war in der That ein überraschender, besonders dann, wenn die Kranken in einem acuten Exacērbationsstadium der gonorrhoischen Entzündung in die Klinik eintraten. Sobald der erste Schmerz, welchen der Leibschnitt als solcher mit sich bringt, am 3. bis 4. Tage post operationem sich gelegt hatte, äusserten die Frauen ihre Freude darüber, dass sie sich zur Operation ,entschlossen hatten; die früheren so heftigen Unterleibsbeschwerden waren vollständig wie mit einem Schlage verschwunden.

Bei der Entlassung aus der Klinik in der 3.-4. Woche wurde dagegen schon oft bei der bimanuellen Betastung eine leichte Druckempfindlichkeit vor Allem in der Gegend der Tubenecken und häufig auch der Ovarien constatirt. Man führte diese Schmerzhaftigkeit auf Stumpfexsudate zurück und beruhigte sich anfänglich mit der Hoffnung, dass dieselben im weiteren Verlauf bald verschwinden würden. Diese Hoffnung erfüllte sich jedoch nicht immer, sondern oft genug steigerten sich die Beschwerden, besonders dann, wenn einige Wochen nach der Operation die Frauen ihre Haus- oder Fabrikarbeit wieder aufnahmen.

Der Uterus war dann oft auf Druck schmerzhaft; links und rechts von ibm hatten sich schmerzhafte und druckempfindliche Stellen ausgebildet; gar nicht so selten traten auch die früher vorhanden gewesenen unregelmässigen Blutungen zwischen den einzelnen Perioden und während der Periode selbst auf; die Cohabitationen waren wieder in gleicher Weise schmerzhaft wie vor der Operation.

Gewiss giebt es Fälle, welche dauernd beschwerdefrei blieben; aber bei der grossen Mehrzahl der Fälle, die ich einige Zeit nach der Operation wieder untersuchen konnte, ist dieses Resultat nicht erzielt; manche Frauen äusserten unverhohlen ihre Unzufriedenheit mit dem Resultat der Operation.

Die Auffassung, dass diese Schmerzen neben dem Uterus durch Stumpfexsudato bedingt sind, hat auch uns dazu geführt, die Asepsis bei der Operation noch mehr zu versehärfen und die Tubenstümpfe möglichst klein zu machen bezw. sie durch Keilexcisionen aus dem Uterus ganz zu entfernen. 
Kroenig, Zur Prognose der ascendirten Gonorrhoe beim Weibe. 393

Der erwünschte Erfolg trat auch jetzt nicht ein. Die Vermuthung, dass es sich um circumscripte Eiterungen um den Stumpf handelte, in Folge secundärer Infection bei der Operation, wurde auch durch die Beobachtung widerlegt, welche man nach Entfernung der Tuben, durch andere Affectionen bedingt, machte. Besonders auffallend erschien mir immer der weitere Verlauf nach Exstirpation der graviden Tube im Vergleich zur Exstirpation der gonorrhoischen Sactosalpinx.

Bei den Patientinnen, welche ich nach Entfernung des graviden Tubensackes einige Zeit post operationem wieder untersuchen konnte, habe ich derartige schmerzhafte Stumpfexsudate niemals constatirt, sondern diese Frauen erfreuten sich meistens eines andauernden Wohlseins, ganz im Gegensatz zu den Frauen nach Entfernung der gonorrhoischen Sactosalpinx.

Die Erfahrungen der Leipziger Klinik stehen nicht vereinzelt da, sondern sind auch gemacht und mitgetheilt worden von vielen anderen Operateuren, besonders von denjenigen, welche über eine grosse Erfahrung verfügen.

Ich erwähne nur einige Aeusserungen von Fritsch, Hegar, Martin, Winter, Landau und die frühzeitigen Berichte von Chrobak und Schauta.

Hegar sagte auf dem Gynäkologen-Congress in Kopenhageu: "Die Erfolge der Operation in Bezug aut die Beschwerden sind aber nicht sehr günstig, da häufig recurrirende Entzündungen nach gut abgelaufenen Operationen selbst noch später nachfolgen können."

Gusserow sagt auf der 59. Naturforscher-Versammlung in Berlin 1886: „Die Indication zur Exstirpation der Tubensäcke hängt im wesentlichen von der Intensität der perimetritischen Beschwerden ab, da die Entfernung der Säcke nicht stets zur Heilung der Beschwerden führt." Auch nach Winter wird nur ein Theil der Kranken durch Salpingotomie gesund, ein anderer Theil wird nur gebessert, und ein nicht unbeträchtlicher Theil - nach seinen Erfahrungen ungefähr die Hälfte - behält die Beschwerden oder wird sogar noch kränker.

Nach A. Martin's ${ }^{1}$ ) Ansicht machen sich nach der Operation noch die Narbenexsudate geltend. Die Prognose ist nach ihm

1) Martin, Discussion über die Adnexoperationen. Gynäliologen-Congress 1893. 
394 Kroenig, Zur Prognose der ascendirten Gonorrhoe beim Weibe.

nicht absolut sicher zu stellen. "Auch wenn die Temperaturkurve einen vollständig normalen, fieberf̣reien Verlauf zeigt, finden wir oft in der 3. Woche bei der Untersuchung Narbenexsudate, die anfänglich allerdings keine Beschwerden machen, später aber wiederum die Quelle neuer schwerer Leiden werden".

Diese Verhältnisse sind nach der Ansicht von Martin bei dem heutigen Stande der Technik nicht zu bessern; wir können die Kranken nicht vollständig davor bewahren. "Dies wirtt einen Schatten auf die Dauerresultate und beeinträchtigt den Werth der Operation erheblich."

Nachdem Wertheim zuerst nachgewiesen und viele Autoren es bestätigt hatten, dass die Gonokokken nicht nur die Schleimhaut der Tuben, sondern auch das Myometrium des Uterus, das Ovarium, das Beckenperitoneum befallen können, lag die Annahme nahe, dass die fortdauernden Beschwerden nach der Operation dureh ein Fortbestehen der Entzündung in diesen Organen bedingt seien. Infolge dessen beschränkte man sich nicht auf die Entfernung der kranken Tube, sondern exstirpirte gleichzeitig beide Ovarien und in letzter Zeit vor allem nach dem Vorgange französischer Autoren auch den Uterus.

Da es sich der Natur der Sache entsprechend bei diesen Erkrankungen hauptsächlich um jugendliche Individuen handelt, so stellte sich bei dieser Operationsweise ein neues, störendes Moment bei den Dauererfolgen ein.

Nach der Exstirpation der Ovarien und des Uterus traten im jugendlichen weiblichen Körper Veränderungen ein, deren pathologische Bedeutung erst allmählich voll erkannt wurde. Es ist Glaevicke's Verdienst, dass er in seiner. Schrift „Körperliche und geistige Veränderungen im weiblichen Körper nach künstlichem Verlust der Ovarien einerseits und des Uterus andererseits" auf diese Erscheinungen nachdrücklichst hingewiesen hat. Jeder Gynäkologe hatte gewiss schon vorher ähnliche Beobachtungen gemacht; aber sie waren nicht in einer so zusammenfassenden Weise dargestellt, wie sie Glaevicke hier giebt. Die Wallungen, Gefühle der heissen Uebergiessung, fliegende Hitze - Erscheinungen, welche oft schon der normalen Climax zugehören - treten hier, in der künstlich auf der Höhe der Geschlechtsreife antecipirten Climax, in viel intensiverer Form und störender Weise auf.

"Schwerer, als diese vorübergehenden Erscheinungen, machen sich die Störungen des psychischen Verhaltens der früh kastrirten 
Frauen bemerkbar; oft finden wir eine gedrückte Gemüthsstimmung, einen schweren melancholischen Zustand; es tritt Verlust des Selbstvertrauens und Lebensmuthes ein. Manehe Frauen werden in ihrer Gemüthsstimmung dadurch so schwer beeinflusst, dass ihnen jetzt nach Cessatio mensium zur klaren Erkenntniss kommt, dass jede Möglichkeit der Fortpflanzung ihnen genommen ist. Das Glück der Ehe wird oft genug weiter dadurch gestört, dass bei der engen, frühsenil-atrophischen Vagina jede Cohabitation unmöglich ist."

In drei Fällen beobachtete Glaevicke ausserdem noch wirkliche Psychosen, welche die Aufnahme in eine Nervenheilanstalt nothwendig machten.

Auch uns in der Leipziger Klinik sind derartige Erfahrungen bei Kastration der Frauen nach gonorrhoischen Adnexerkrankungen nicht erspart geblieben; wir haben sie im allgemeinen aber nur selten gemacht, weil $\mathrm{Zweifel}$ bei Frauen auf der Höhe der Geschlechtsreife auf Grund dieser Erfahrungen nur relativ selten sich zur Exstirpation des Uterus und seiner Anhänge wegen dieser Erkrankung entschliessen konnte.

Bei Frawen nahe der Climax treten diese erwähnten Ausfallserscheinungen seltener und nur wenig störend auf, hier werden in der That durch das radikalere operative Vorgehen bei den gonorhoischen Adnexerkrankungen die Erfolge der Operation gebessert. Da diese Fälle selbstverständlich nur die Minderzahl bildeten, so konnte anch jetzt noch von einem den Operateur befriedigenden Erfolge nach der Operation im allgemeinen nicht die Rede sein. Fritsch hat nemerdings erst wiederum auf der Naturforscher-Versammiung in München es klar ansgesprochen, wie wenig dankbar die operativen Erfolge bei den gonorrhoischen Adnexerkrankungen auch zur Zeit noch sind:

Dies war der Grund, welcher $Z$ weifel veranlasste, in allen Fällen von gonorrhoischen Adnexerkrankungen methodisch das operative Verfahren abzulehnen, um so ein Bild zu erhalten, wie sich selbst in den anfänglich schwersten Formen der ascendirten Gonorrhoe der weitere Verlauf gestaltet.

Hiermit wurde gleichzeitig dem Einwand begegnet, den man bei früheren Untersuchungen über die klinische Bedeutung der ascendirten Gonorrhoe machen konnte, dass man hauptsächlich Fälle mit an und für sich schon günstiger Prognose in ihrem 
396 Kroenig, Zur Prognose der ascendirten Gonorrhoe beim Weibe.

klinischen Ablauf beobachtet hatte, während die schwereren Fälle operativ behandelt wären.

Den nachfolgenden Untersuchungen kam weiter zu statten, dass die Beobachtungen an Fraven der arbeitenden Klasse gemacht wurden, welche also meistens nur wenig körperliche Schonung sich gestatten konnten, sodass auch von einer Auswahl günstiger Fälle nicht zu sprechen war.

Die specielle Veranlassung zu nachfolgender Zusammenstellung gab eine über die Prognose der gonorrhoischen Erkrankungen geäusserte Ansicht unseres ersten Forschers in der Gonorrhoefrage, Bumm, ${ }^{1}$ ) welcher bei seiner letzten zusammenfassenden Bearbeitung der gonorrhoischen Erkrankungen der weiblichen Harn- und Geschlechtsorgane sich im Handbuch der Gynäkologie von Veit folgendermaassen ausspricht:

"Die Heilungsaussichten der Tubengonorrhoe - wenigstens was die allgemeine Gesundheit, Leistungs- und Genussfähigkeit der Erkrankten betrifft - hat man meines Erachtens in der neueren Zeit zu ungünstig hingestellt. Auch da, wo die Infection bis in die Tuben forgeschritten ist, sind die Fälle mit Ausbildung grosser Eitersäcke und stetig wiederkehrenden Anfällen der Pelveoperitonitis in der Minderzahl und es verdient allzu operationslustigen Aerzten gegenüber hervorgehoben zu werden, dass mit der Feststellung einer gonorrhoischen Salpingitis poch nicht alles verloren ist, sondern bei geeignetem Verfahren und Geduld sehr wohl eine Ausheilung bis zu einem fast beschwerdefreien Zustand nicht nur möglich ist, sondern viel öfter vorkommt, als man eine Zeit lang geglaubt hat."

Es sollte versucht werden, an dem gegebenen Materiale diese hier niedergelegte Ansicht zu prüfen. Zu diesem Zwecke wurden die Frauen aus dem Zeitraum, wo die gonorrhoischen Adnexerkrankungen in der Leipziger Klinik principiell nicht operativ behandelt wurden, zur. Nachuntersuchung wieder bestellt. In der grössten Mehrzahl der Fälle waren es Patientinnen, welche eine Zeit lang klinisch beobachtet waren; nur einige wenige Fälle waren ausschliesslich in der Poliklinik behandelt.

Bei der Nachuntersuchung von Fällen, bei denen die Diagnose

1) Bamm, Die gonorrhoischen Erkrankungen der weiblichen Harn- und Geschlechtsorgane. Handbuch der Gynäkologie von Veit. Wiesbaden. Verlag von J. F. Bergmann. 1897. 1. Bd. S. 501. 
Kroenig, Zur Prognose der ascendirten Gonorrhoe beim Weibe. 397

nicht durch die Operation bestätigt ist, ist natürlich zunächst zu erwägen, ob von vornherein eine irrthümliche Diagnose mit genügender Sicherheit vermieden werden kann.

Uns leiteten bei der Diagnose der gonorrhoischen Adnexerkrankung, vor allem der gonorrhoischen Saktosalpinx, die Gesichtspunkte, welche in so überaus klarer Weise Winter in seiner "gynäkologischen Diagnostik" auseinandergesetzt hat.

Wichtig war uns vor allem das Palpiren eines derben Stranges des "Tubenstrangs", welcher vom Uterushorn zum Tumor sich hinzieht. Dieser Strang ist die derbinfiltirte Wand der. Tube, welche meistens in Narkose am Uterushorn sehr deutlich durchzutasten ist. Wir müssen nach unseren Erfahrungen Winter darin beipflichten, dass der Nachweis dieser Verdickung am uterinen Theile der Tube als das sicherste Zeichen eines Tubentumors anzusehen ist.

Weiter war uns die Doppelseitigkeit der Affection massgebend. Wir haben nur bei denjenigen Tumoren gewagt, die Diagnose auf gonorrhoische Pyosalpinx zu stellen, bei welchen diese Doppelseitigkeit klinisch durch den Tastbefund sicher constatirt werden konnte. Ein vollständiges Freibleiben der einen Seite bei deutlich ausgeprägter Veränderung der anderen Seite gehört bei den gonorrhoischen Adnexerkrankungen zu den grössten Seltenheiten; die Doppelseitigkeit ist auch klinisch durch den Tastsinn meistens oder fast immer nachweisbar. Allerdings darf man gleichgrosse Tumoren auf beiden Seiten hier nicht erwarten; dièse findet man nur in einer beschränkten Anzahl der Fälle. Wohl aber konnten wir, wenn wir auf der einen Seite einen deutlich ausgebildeten Tumor constatirten, meistens auf der anderen Seite wenigstens eine deutlich verdickte Tube feststellen, welche oft mit dem Ovarium und mit der Beckenwand durch breite Adbäsionen verbunden war.

Martin hat allerdings bei Erkrankungen der Tube neben 525 doppelseitigen Erkrankungen 959 einseitige zu notiren gehabt; nach ihm legt die Doppelseitigkeit der Erkrankung vielleicht die Vermuthung eines tubaren Ursprungs nahe, sie giebt aber keinen sicheren Anhaltspunkt weder für den Ursprung der Geschwulst - ob ovarial oder tubar -- noch für seinen Inhalt.

Bei den zur Operation gekommenen gonorrhoischen Tubenerkrankungen haben wir in der Leipziger Frauenklinik eine derartige Erfahrung nicht machen können, und es ist möglich, dass die Differenz dadurch bedingt ist, dass Martin nicht ausschliesslich auf die Tubenerkrankungen gonorrhoischen Ursprungs zurück- 
greift. Dies ist auch von Martin nicht besonders betont, sodass die Ansicht von Martin derjenigen Winter's und unserer nicht widerspricht.

Zur Diagnose verlangten wir weiter bei unseren Fällen eme nachweisbare perimetritische und pelveoperitonitische Entzündung, welche sich durch Schmerzhaftigkeit des Uterus, Verwachsung und Adhäsionsbildang des Uterus und seiner Adnexe mit dem Beckenboden und den Darmschlingen geltend machte. Diese entzündlichen Complicationen kömnen bei anderen Erkrankungen, bei Geschwülsten des Eierstockes, des Uterus, z. B. bei subserösen Myomen manchmal ebenfalls vorhanden sein, doch treten sie in dieser diffusen Ausdehnung und so deutlich klinisch nachweisbar, wie gerade bei den gonorrhoischen Tubenentzündungen selten auf.

Leider waren wir in der Mehrzahl unserer Fälle nicht imstande, ausser diesen Symtomen gleichzeitig einen gonorrhoischen Cervixkatarrh oder eine noch bestehende gonorrhoische Entzündung der Urethralschleimhant und der Ausführungsgänge der Bartholinschen Drüsen festzustellen. Der Nachweis von Gonokokken im Cervixsekret ist nur in 2 wei der mitzutheilenden Fälle erbracht worden. Manchmal áber konnten wir die von Saenger erwähnten concomittirenden Röthungen und Verfärbungen am Scheideneingang, vor allem an den paraurethralen Gängen und den Ausführungswegen der Bartholinschen Drüsen mit zur Diagnose verwerthen.

Die Probepunction ist nur in ganz vereinzelten Fällen wegen der ihr anhaftenden Gefahr der Infection auch bei peinlichster Handhabung der Asepsis und der Gefahr der Darmverletzung ausgeführt worden. Sie hat bei der gonorrhoischen Adnexerkrankung deswegen um so weniger Werth, weil man oft genug mit der Punktionsnadel, die man vom hinteren Scheidengewölbe aus vorstösst, gar nicht den Eiter in der Tube trifft, sondern die, den entzündlichen gonorrhoischen Process so häufig begleitenden Serocelen, sodass man bei Entleerung von klarer Flüssigkeit eher noch durch einen derartigen Befund irre geleitet werden könnte.

Parametritische Entzündungen haben wir niemals bei der gonorrhoischen Adnexerkrankung als Begleiterscheinung klinisch feststellen können; ja wir haben bei einem deutlichen klinischen Nachweis einer Parametritis die Diagnose auf gleichzeitige gonorrhoische Entzündung der Adnexe, selbst wenn sonstige Symptome vorbanden waren, fallen lassen. Wir möchten den Satz Winter's in sejner "gynäkologisehen Diagnostik", dass die Parametritis 
Kroenig, Zur Prognose der ascendirten Gonorrhoe beim Weibe. 399

sclten eine Begleiterin der Tubenerkrankung ist, dahin modificiren, dass eine klinisch nachweisbare Parametritis nie die Tubenerkrankung begleitet.

Die Anamnese stützte in den meisten unserer Fälle bis zu einem gewissen Grade ebenfalls die Diagnose. Wenn auch die Aussagen der Frau mit Vorsicht aufgenommen werden müssen, so sind doch Angaben wie, Entstehung eines eitrigen Fluors kurz nach der Verheirathung oder einige Zeit nach dem Wochenbett, häufiges Brennen beim Wasserlassen, erneutes Auftreten von Schmerzattaquen, besonders nach Cohabitationen and nach schweren körperlichen Anstrengungen, gute Wegweiser.

Immerhin mag - und wir leugnen dies keineswegs - unter den angeführten Fällen auch manche Fehldiagnose sein. Doch sind wir heute eher berechtigt, eine gestellte Diagnose auf gonorrhoische Tubenerkrankwug als gesichert zu betrachten, weil unsere Diagnose durch die combinirte Untersuchung, vor Allem aber durch die häufige Controle des Palpationsbefundes bei der Laparotomie eine ungleich viel grössere Feinheit und Zuverlässigkeit gewonnen hat wie früher. Gerade die so häufig auch in der Leipziger Frauenklinik bei gonorrhoischen Adnexerkrankungen in früherer Zeit ausgeführten Operationen haben uns gleichzeitig Gelegenheit gegeben, die Sicherheit unserer Diagnose durch den Befund bei der Operation zu prüfen. Als Assistent der Leipziger Frauenklinik hatte ich längere Zeit die gynäkologische Aufnahmestation und war infolge dessen in der Lage, die vor der klinisehen Demonstration gestellte Diagnose mit dem Befund bei der später ausgeführten Laparotomie zu vergleichen. Auf Anregung von $\mathrm{Zweifel}$ wird am Ende jedes Semesters eine Statistik angefertigt über die Anzahl der Fehldiagnosen, welche bei intraabdominell gelegenen Tumoren gemacht sind in den Fällen, wo später die Diagnose durch die Laparotomie klargelegt werden konnte. Diese Statistik hat den merkwürdigen Befund ergeben, dass in den letzten acht Jahren die Zahl der Fehldiagnosen ungefähr stets auf gleicher Höhe geblieben ist, nämlich circa $8 \mathrm{pCt}$. der Diagnosen überhaupt, bald etwas weniger, bald auch wieder etwas mehr; natürlich sind hier nur diejenigen Diagnosen gerechnet, bei welchen es sich um rein intraabdominelle Tumoren handelt, also Carcinome des Uterus, submucöse Myome etc., welche durch Dilatation des Cervicalcanals direct getastet werden konnten, sind hier nicht mitgerechnet.

Die genauere Analyse der einzelnen Fehldiagnosen ergab aber 
400 Kroenig, Zur Prognose der ascendirten Gonorrhoe beim Weibe.

weiterhin, dass gerade bei den gonorrhoischen Adnexerkrankungen die Diagnose in den seltensten Fällen verfehlt worden ist. Das Fühlen des Tubenstranges, die Doppelseitigkeit der Erkrankung, die breiten Adhäsionen der Tumoren mit der Beckenwand bieten uns so wesentliche Stützpunkte der Diagnose, dass wir hier selten ausgleiten. Die 8 pCt. in der Statistik sind hauptsächlich bedingt durch Fehler bei der differentiellen Diagnose zwischen Cystomen and erweichten Myomen, zwischen Ovarialerkrankungen und Extrauteringravidität.

Wenn wir daher auch zugeben, dass unter den mitzutheilenden Fällen manchmal eine Fehldiagnose mit untergelaufen ist, so ist doch immerhin die Wahrscheinlichkeit eine sehr grosse, dass es sich bei den 38 Fällen nur um einige wenige Fälle gehandelt, hat, und dass wir die Mehrzahl dieser heute nach der Verfeinerung der Diagnose entschieden als richtig diagnosticirte gonorrhoische Anexerkrankungen ansehen dürfen.

Diese heute so sehr viel gesichertere Diagnostik der ascendirten Gonorrhoe erhöht den Werth einer weiteren Beobachtung dieser Fälle, und liess mir die hierbei gemachten Beobachtungen der Veröffentlichung werth erscheinen.

Im Ganzen konnten 38 Fälle nachuntersucht, bezw. brieflich von ihnen Nachricht erhalten werden. Ich gebe nicht alle 38 Krankengeschichten ausführlich wieder, sondern nur einen Theil derselben (21), und zwar vornehmlich diejenigen, bei denen ein ausführlicher Aufnahmestatus vorhanden war, vor allem aber auch noch ein gynäkologischer Tastbefund bei der Nachuntersuchung erhoben werden konnte. Einige von den 38 Frauen theilten nämlich nur brieflich ihr Befinden mit, verweigerten dagegen, weil sie keine Beschwerden mehr hatten, jede ärztliche Untersuchung.

Die Schlussfolgerungen sind, soweit dies möglich war; auf sämmtlichen 38 Fällen basirt. Am Schluss der Arbeit sind die Krankengeschichten in Kürze wiedergegeben.

Wie schon erwähnt war die grösste Zahl der nachuntersuchten Fälle (27) kürzere oder längere Zeit in klinischer Behandlung gewesen; es sind diejenigen, bei denen die acuten Erscheinungen die Frau arbeitsunfähig gemacht hatten, und bei denen eine häusliche Pflege unmöglich war.

Bei den 27 Fällen, welche klinisch behandelt wurden, war der Aufenthalt in der Klinils gewöhnlich ebenfalls nur ein vorübergehender, und zwar deswegen, weil die acuten Erscheinungen durch 
die angewandte conservative Therapie so schnell zurückgingen, dass die Pat. bald, wieder ihre Arbeit aufnehmen konnten. Die längste Verpflegungszeit haben wir im Fall 1 mit 38 Tagen; dann folgen 2 Fälle mit einer klinischen Verpflegungszeit von 25 und 24 Tagen. Alle übrigen Fälle sind wesentlich kürzer in Behandlung gewesen, im Durchschnitt 12 bis 14 Tage. Bei einigen Fällen dauerte der Aufenthalt in der Klinik nur wenige Tage, z. B. in einem Falle 2, in fünf Fällen 4 Tage, und in vier Fällen 9 Tage.

Elf Fälle wurden überhaupt nicht in klinische Behandlung aufgenommen, sondern poliklinisch behandelt.

Die Behandlung in der Klinik war gewöhnlich folgende: Bestand neben sehr starken Schmerzen Fiebersteigerung, so wurden zunächst Abführmittel, und zwar vornehmlich die Salina gereicht, ausserdem wurde eine Eisblase auf das Abdomen gelegt. Eine locale Behandlung von der Scheide aus fand dagegen zunächst nicht statt. Nach Entleerung des Darmes, welche meistens so weit getrieben wurde, dass die ersten Tage 4-6 mal Stuhlgang pro die erfolgte, liess das Fieber in allen Fällen nach, und zwar gewöhnlich in wenigen Tagen. Bei Bettruhe der Patientinnen trat in den meisten Fällen Nachlass der heftigsten Beschwerden ein. Sobald die Beschwerden sich gebessert hatten, wurden abwechselnd Einlagen mit Ichthyoltampons in die Scheide gemacht, oder heisse Scheidendouchen verordnet, und zwar in der Weise, dass an einem Tage die Einlagen gemacht wurden und am folgenden Tage zweimal täglich eine heisse Scheidenspülung bis zu $40^{\circ} \mathrm{O}$. Die stärkeren Blutungen veranlassten uns in mehreren Fällen Stypticin dreimal täglich in Dosen von $0,05 \mathrm{~g}$ zu reichen, worauf die Blutung meistens sehr bald sistirte. Während der Behandlung in der Klinik wurde, um compakteren Stuhl zu vermeiden, nur flüssige Diät den Kranken verabreicht; auch hierbei wurde darauf gesehen, dass gleichzeitig durch die Wahl der Speisen eine leicht purgirende Wirkung erzielt wurde.

Bei der Entlassung aus der klinischen Behandlung war keineswegs immer eine schon vollständige Genesung erzielt, sondern es wurden die Frauen meistens dann entlassen, wenn die Beschwerden sich so weit gebessert hatten, dass die Frauen wenigstens ihre häusliche Arbeit wieder aufnebmen konnten.

Bei Patientinnen. der Ortskrankenkasse wurde meistens darauf gesehen, dass dieselben noch 2-3 Wochen nach Entlassung aus der Klinik arbeitsunfähig geschrieben wurden. 
402. Kroenig, Zur Prognose der ascendirten Gonorrhoe beim Weibe.

Bei den Pat., welche nur poliklinisch hehandelt wurden, wurde im Allgemeinen nach denselben Principien verfahren, nor mit dem Unterschiede, dass nicht in gleichcorrecter Weise die einzelnen Massnahmen ausgeführt werden konnten. Es scheiterte einmal an der einzuhaltenden Bettruhe, vor Allem aber auch häufig an dem Geschlechtstrieb des Mannes, welcher trotz energischen Verbotes weitere Cohabitationen ausführte. Dieser Umstand stellt in der Behandlung der gonorrhoischen Adnexerkrankungen einen vie] störenderen Factor dar, als die zu frühe Aufnahme körperlicher Arbeit.

Auch die poliklinische Behandlung dauerte in den meisten. Fällen nicht länger als einige Wochen, einmal deswegen, weil die Frauen ihre Beschwerden verloren hatten, dann aber auch sicherlich in einigen Fällen, weil die häushiche Arbeit oder die wieder aufgenommene Fabrikarbeit das häufigere Besuchen der poliklinischen Sprechstunde unmöglich machte. -

Betrachten wir zunächst die objectiven durch den Genitaltastbefund nachweisbaren Veränderungen, so waren in der grössten Zahl der untersuchten Fälle ziemlich ansehnliche Tumoren bei der ersten klinischen Untersuchung neben dem Uterus getastet. Wir finden z. Th. Tumoren bis zu Emueigrösse zu beiden Seiten des Uterus und hinter demselben liegend bei dem Aufnahmebefund erwähnt.

Fast überall ist schon nach wenigen Tagen der Behandlung, besonders wenn die Frauen stark purgirt hatten und strenge Bettruhe beobachtet hatten, während des klinischen Aufenthaltes eine Verkleinerung des Tumors constatirt, welche in manchen Fällen so auffallend ist, dass man zunächst an einen falschen Tastbefund bei der ersten Untersuchung glauben möchte.

Diese schnelle Verkleinerung kann ihre Erklärung in verschiedener Weise finden.

Es ist wohl kaum annehmbar, dass eine eitrige Sactosalpinx so schnell an Volumen durch Eindickung des Eiters oder Resorption desselben abnimmt, wie es dieser Verkleinerung der Tumoren entspricht. Es ist vielmehr wahrscheinlich, dass die Volumabnahme der Tumoren in erster Linie dadurch bedingt ist, dass die Serocelen, welche die Sactosalpinx fast stets begleiten, schwinden. Diese abgekapselten, intraperitonealen serösen Ergüsse können, wie jeder Operateur bestätigen wird, neben kleinen Pyosalpinxsäcken den grössten Theil des getasteten Tumors ausmachen. Es ist aber wohl möglich, dass diese entzündliche Aus- 
Kroenig, Zur Prognose der ascendirten Gonorrhoe beim Weibe. 403

schwitzung durch Bettruhe und antiphlogistische Behandlung sehr schnell resorbirt wird.

Weiter kommt in Betracht eine Uebersehätzung der Grösse des eigentlichen Tumors dadurch, dass die adhärenten Därme bei der bimanuellen Betastung mit zu dem Tumor gerechnet werden. Die Pyosalpinxsäcke sind fast stets mit Darmschlingen und speciell mit der Flexura sigmordia verwachsen; sind bei der bestehenden Obstipation diese Darmtheile mit Koth prall gefüllt, so ist es dem Tastsinn nicht möglich, diese gefüllten Därme bei ihrer innigen Adhärenz von dem eigentlichen Pyosalpinxsack scharf zu trennen; die pelveoperitonitischen Adhäsionen erschweren die Abgrenzung der Sactosalpinx gegen die Umgebung ausserordentlich. Werden jetzt durch die dargereichten Abführmittel die Därme geleert, so erscheint uns bei der bimanuellen Betastung der Pyosalpinxsack wesentlich verkleinert, während die Verkleinerung in aller erster Linie dureh die Entleerung der adhärenten Darmabschnitte bedingt war.

In einer grossen Zahl unserer Fälle war es 'uns möglich, bei dén zur Nachuntersuchung wiederbesteliten Kranken von Neuem einen Genitaltastbefund aufzunehmen; nachdem die Franen schon lange aus der klinischen und poliklinischen Behandlung entlassen waren.

In keiném Fall ist in dem Zeitintervall von ungefähr 3 bis 4, Jahren nach der ersten Untersuchung eine wirkliche restitutio in integrum derart, dass wir es mit einem vollständig normalen Genitaltastbefund zu thun haben, eingetreten.

Ueberall konnte entweder eine Verdickung der Tuben am uterinen Ende oder eine Verwachsung des' wenig vergrösserten Ovariums auf dem Beckenboden noch festgestellt werden. Manchmal sind sogar noch ganz ansehnliche Tumoren neben dem Uterus zu fühlen, welche allerdings meistens auf Druck unempfindlich sind.

Um jede subjective Beeinflussung auszuschalten, habe ich die Nachuntersuchung von andererSeite ausführen lassen. Dr. Mattiesen, damaliger Assistent der Klinik, hatte die grosse Freundlichkeit, die einzelnen Fälle, ohne dass ich ihm irgend etwas vorher sagte, zu untersuchen und den aufgenommenen Gènitaltastbefund zu controliren.

Die Nachuntersuchung bei den Pyosalpinxsäcken hat weiterhin gelehrt, dass durch die Einschmelzung der hinter dem. Uterus gelegenen Tumormassen der anfänglich in Anteversio-flexio liegende Uterus nachträglich in Retroversio-flexio sich befand, und zwar fest 
404 Kroenig, Zur Prognose der ascendirten Gonorrboe beim Weibe.

an der hinteren Beckenwand fixirt. Es ist dies eine natürliche Folge der an Stelle des Tumors getretenen Narbe bezw. Adhäsion. Von 24 Fällen, bei welchen nachträglich, gewöhnlich 3 Jahre nach der ersten Schmerzattacke, von neuem ein gynäkologischer Tastbefund erhoben werden konnte, war der Uterus in 12 Fällen retrovertirt oder -flectirt und breit hinten fixirt, sodass es nicht gelang, denselben durch Druck von der Scheide aus oder durch combinirtes Verfahren von den Bauchdecken und von der Soheide aus in Anteversion zu bringen.

In einem. Fall war die Haltung des Uterus noch eine anteflectirte, aber der Uterus war in toto retroponirt und in seinem unteren Theile durch Verödung des Douglas'schen Raumes binten an die Beckenwand breit fixirt.

In mehreren Fällen konnten neben dem retrofixirten Organe noch entzündliche, auf dem Beckenboden'verwachsene Tumoren von Hühnerei- bis Gänseeigrösse festgestellt werden, welche auf Druck meistens nicht schmerzhaft waren.

In 11 Fällen lag der Uterus bei der Nachuntersuchung in Anteversio-flexio und war bei der bimanuellen Betastung in den meisten Fällen auch ziemlich beweglich. Eine vollständige Unbeweglichkeit des Uterus durch Adhäsionsbildung ist hierbei nur in einem Falle notirt. Auch bei antevertirtem Uterus sind mehrmals noch entzündliche Adnextumoren neben dem Uterus bei Aufnahme des Genitaltastbefundes festgestellt,

In manchen Fällen beschränkte sich das Anormale des Genitaltastbefundes allein auf eine Fixation des wenig vergrösserten Ovariums bezw. auf eine leichte Schwellung der Tuben.

In keinem unserer 38 Fälle, trotzdem bei allen nach Aussage reichlich Gelegenheit zur Conception vorhanden war, ist jemals wieder eine Empfängniss eingetreten. Die Verwüstung, welche die Gonokokken in den Anhängen der Gebärmutter ausüben, scheint in der That eine irreparable za sein, wie dies ja auch schon von anderen Autoren behauptet ist.

Besonders charakteristisch dafür, wie sicher die gonorrhoischen Adnexerkrankungen Sterilität erzeugen, ist der Fall No. 12 (im Anhang), wo bei der 31 jährigen Frau, die bis zur Erkrankung 12 normale Entbindungen schnell hintereinander durchgemacht hatte; nach der nachgewiesenen gonorrhoischen Affection der Anhänge trotz dreijähriger Beobachtung weder eine Fehlgeburt noch eine Geburt erfolgte. 
Etwas Aehnliches sehen wir im Fall 11. Hier waren bei der 30jährigen Frau 5 Entbindungen schnell aufeinander vorausgegangen. Die Gonorrhoe ascendirte nach der letzten Entbịndung im Spätwochenbett in die Tuben; seit der Zeit ist in der dreiiährigen Beobachtungszeit keine Conception wieder erfolgt.

Immerhin darf heute noch nicht nach einer so kurzen Beobachtungszeit von 3-4 Jahren ein definitiver Entscheid gegeben werden. Es wäre an und für sich denkbar, dass sich auch schwer erkrankte Tuben im Verlaufe von mehreren Jahren vollständig wieder erholen und der etwa verschlossene Pavillon der Tube sich wieder öffnet. Gerade bei den Fällen, in denen der Uterus bei der Nachuntersuchung nach dem dreijährigen Intervall beweglich vorn gefühlt, und in denen nur bei genauester Erhebung des Tastbefundes noch eine Verdickung der Tuben festgestellt. werden konnte, ist eine nachfolgende Conception und ein günstiger Ablauf der Schwangerschaft im wieder beweglich gewordenen Uterus keineswegs an und für sich ausgeschlossen.

Wir werden im Weiteren sehen, wie nach einem als gonorrhoisch nachgewiesenen pelviperitonitisehen Exsudate hinter dem Uterus später noch, allerdings nach vielen Jahren, Conception eintreten kann und die narhfolgenden Geburten einen vollständig normalen Verlauf nehmen.

Das subjective Befinden der Frauen mit gonorrhoischen Adnexerkrankungen nach der Entlassung aus der klinischen oder poliklinischen Behandlung war natürlich bei den einzelnen Frauen verschieden, und zwar verschieden, je nachdem die Frauen in der Lage waren, sich körperlich zu schonen, vor Allem aber auch verschieden danach, ob die Frauen vor einer erneuten gonorrhoischen Infection einigermaassen geschützt waren. Von 38 beobachteten und nachuntersuchten Fällen sind die meisten so weit gebessert worden, dass sie schon bald nach den ersten heftigen Schmerzattacken nach der klinischen Behandlung ihre Arbeit wieder vollständig aufnehmen konnten. Wenn auch vorübergehend noch manchmal acute, heftigere Beschwerden auftraten, so ist doch im Allgemeinen von diesen reeidivirenden Attacken zu sagen, dass, je länger die erste Erkrankung zurückliegt, desto geringer und schneller ablaufend die einzelnen Schmerzanfälle sind, sodass die Patientinnen oft genug deswegen gar nicht wiederum den Arzt aufsuchten, sondern mit den durch eigene Erfahrung erprobten Mitteln (vorübergehende Bettruhe, Sitzbäder) sich begnügten. 
406 Kroenig, Zur Prognose der ascendirten Gonorrhoe beim Weibe.

Die. genauere Analyse der Beschwerden bei den 38 Fällen ergiebt Folgendes.

Von den 38 Frauen nahmen nach der erstmaligen klinischen oder poliklinischen Behandlung 15 Frauen nachträglich keine weitere ärztliche Behandlung in Anspruch. 8 Patientinnen blieben noch einige Wochen in poliklinischer Behandlung oder gingen in anderweite ärztliche Behandlung über; doch waren es nicht immer Sehmerzen, sondern : oft noch der länger andauernde Ausfluss, welcher sie veranlasste, ärztliche" Hülfe zu suchen. 'Länger dauernde nachträgliche poliklinisché Behandlung bedurften drei Kranke, in einem Falle bis zu einem Jahr.

Bei zehn Patientinnen wurde mehrere Monate nach der ersten Attacke nochmals ärztliche Behandlung nothwendig wegen der-erneuten Schmerzattacken, und in einem Falle zweimalige Aufnahme ins Krankenhaus. Wegen unregelmässiger Blutungen wurde in zwei Fällen vom Arzt später noch eine Abrasio mucosae ausgeführt.

Zur Zeit der Nachuntersuchung, also im Allgemeinen drej: Jahre nach der ersten klinischen Behandlung waren voll arbeitsfähig, d. h. konnten vollständig häusliche und Fabrikarbeit. verrichten von 38 Framen 32. Von den übrigen 6 schaltet eine, später zri erwähnende Puella publica aus. Von den restirenden 5 erklärten 3 , dass sie leichte Arbeit wohl gut verrichten könnten, dass sie aber bei schweren Arbeiten, besonders bei Feldarbeit, noch manchmal Schmerzen im Unterleib empfätiden. Eine Frau klagte darüber, dass sie durch die manchmal ganz unvermuthet eintretenden Schmerzattacken noch häufig an der Arbeit verhindert sei, während die letzte durch: die immerwährenden Beschwerden im Unterleib sich in ihrer Arbeitskraft wesentlich beeinträchtigt sah.

Von 38 Frauen gaben 30 bei der Nachuntersuchung an, dass sie nach der Entlassung aus der Klinik ihre Arbeit gleich wieder aufgenommen, bezw. bei poliklinischer Behandlung ihre Arbeit überhaupt nicht unterbrochen hätten.

Bei den übrigen 8 Frauen scheidet auch hier wiederum die Puella publica aus. Von den restirenden 7 gaben 3 an, dass sie nach einem Zeitintervall von 3-6 Wochen ihre Hausarbeit wieder verrichten konnten, während die übrigen 4 in der Folgezeit oft noch durch die recidivirenden Schmerzanfälle längere Zeit an ihrer Arbeit verhindert wurden.

Den schwersten klinischen Verlauf nimmt, wie wir wissen, die ascendirte Gonorrhoe bei den Prostituirten, da bei ithen eine 
Kroenig, Zur Prognose der ascendirten Gonorrhoe beim Weibe. 407

Ruhigstellung des Uterus und Schonung in sexueller Hinsicht vollständig ausgesehlossen ist. Ich habe nur eine Prostituirte unter den Fällen weiter verfolgen können; es ist dies dadurch bedingt, dass der Wohnungsweehsel bei diesen Personen gewöhnlich ein sebr häufiger ist, so dass es schwer ist, selbst durch das Einwohnermeldeamt ihren Aufenthaltsort $z u$ ermitteln.

In diesem Falle ist das Resultat der Nachuntersuchung als ein sehr schlechtes zu bezeichnen. Patientin hat seit ibrer ersten Behandlung in der Klinik sich wiederholt ins Krankenhaus anfnehmen lassen müssen, um sich dort ebenfalls specialistischer Behandlung zu unterziehen. Auch in der Zwischenzeit sind die Beschwerden niemals ganz verschwunden gewesen. Die Schmerzen bei der Cohabitation traten so heftig auf, dass ihr schliesslich jeder geschlechtliche Verkehr unmöglich wurde.

Vorübergehende Cohabitationen, wie sie'der eheliche Verkehr mit sich bringt, scheinen dagegen einen bei weitem nicht so ungünstigen Einfluss auszuüben, was wohl dadurch bedingt ist, dass eine nene acute gonorrhoische Infection mit virulenten Gonokokken selten erfolgt. Die meisten der verheiratheten Frauen geben an, dass sie sehr bald nach der Entlassung den Geschlechtsverkehr wieder begonnen und keine wesentlichen Beschwerden bei demselben empfunden hätten.

Die Serie dieser nachuntersuchten Fälle führt uns daher zu einer Bestätigung der von Bumm schon oben erwähnten Behauptung; dass das Krankheitsbild der ascendirten Gonorrhoe selbst.dann, wenn die Gonokokken in den Tuben und auf dem Beckenbauchfell tastbare Veränderungen erzeugt haben, ehtschieden etwas zu ungünstig hingestellt ist.

Wenn wir aus 38 Fällen generalisiren dürfen, s $\phi$ ist die grösste Zahl aller Fälle von gonorrhoischer Adnexerkrankung selbst dann, wenn die Frauen der arbeitenden Klasse angehören, in nicht zu langer Zeit wieder so weit hergestellt, dass sie durch die Unterleibsschmerzen nicht wesentlich in ihrer Arbeitsfähigkeit behindert werden. Immerhin bleiben einige, bei welchen jahrelang die Beschwerden anhalten, so dass wir sicherlich troty des im Allgemeinen günstigen Verlaufs auch heute noch nicht auf eine operative Behandlung vollständig Verzicht leisten werden.

Der ungünstige Einfluss, den die bis in die Tuben ascendirte Gonorrhoe in Bezug auf die Fruchtbarkeit des Weibes ausübt, liess die Frage nabe legen, ob auch schon beim Ascendiren 
408 Kroenig, Zur Prognose der ascendirten Gonorrhoe beim Weibe.

der Gonokokken in die Uterushöhle sich ein derartiger Einfluss geltend macht. Bumm glaubt, dass Noeggerath und Glünder die Bedeutung der Gonorrhoe für die Sterilität überschätzt haben. Glünder fand unter 87 sterilen Ehen $62 \mathrm{mal}$ als Aetiologie Tripperinfection $=71,3$ pCt. Bumm berechnete einen viel geringeren Procentsatz; eine Zusammenstellung von 110 Fällen von Sterilität ergab ihm, selbst wenn er die zweifelhatten Fälle noch mitrechnete, nur 30 pCt. durch Gonorrhoe bedingte Unfruchtbarkeit, eine Zahl, die allerdings immer noch gross genug ist.

Das Ascendiren der Gonokokken von den tieferen Theilen des Genitalcanals in höher gelegene findet, wie wir nach den Untersuchungen von Sänger, Bumm, Menge und Verfasser wissen, vornehmlich im Wochenbett statt; nach Bumm bildet sonst der innere Muttermund gewöhnlich eine Grenze für die Ausbreitung der Gonokokken nach oben hin. Die Infection der Corpushöhle im Frühwochenbett von dem gonorrhoisch inficirten Cervikalcanal aus können wir gewissermaassen als das erste Stadium der ascendirten Gonorrhoe betrachten, weil nach Ueberschreitung des inneren Muttermundes die Gonokokken oft in einem Zuge bis in die. Tuben und auf das Beckenbauchfell vordringen.

Schon in einer früheren Arbeit über den Bakteriengehalt des Lochialsecrets der Wöchnerinnen hatte ich gezeigt, wie bei den gonorrhoisch inficirten Frauen in den ersten Tagen post partum eine plötzliche acute Steigerung des Infectionsprocesses statthat, and wie hierbei die Gonokokken sich in grosser Zahl in der Uterushöhle nachweisen lassen. Ich hatte in den Jahren 1893 und 1894 bei einer grösseren Zahl von Wöchnerinnen im Frühwochenbett diese Infection der Uterushöhle in der Weise festgestellt, dass ich im Döderlein'schen Capillarröhrchen, im sogenannten Lochienröhrchen, aus der Uterushöhle direct Secret entnommen und in dem Secret entweder bakteriologisch durch Aussaat des Secrets auf eine Mischung von Agar mit Kystomflüssigkeit (Menge) die Gonokokken festgestellt oder wenigstens mikroskopisch nachgewiesen hatte. Bei dem mikroskopischen Nachweis der Gonokokken gebrauchte jch die Vorsicht, dass stets gleichzeitig eine Aussaat des entnommenen Secrets auf gewöhnliches Agar vorgenommen wurde, umi andere Kokkenarten, die auf Agar ihr Wachsthum finden, wie z. B. den Streptococcus pyogenes und den Staphylococcus pyogenes aureus auszuschliessen, and dass jedesmal das Präparat auch nach Gram gefärbt wurde. 
Diese Vorsicht ist nothwendig, weil diese eben erwähnten Kokkenarten ebenfalls innerhalb der Leukocyten des Lochialsecrets zu. finden sind, und sich durch nichts im Aussehen von Gonokokken unterscheiden.

Ich beauftragte den Cand. med. Lampe, diese sämmtlichen Wöchnerinnen, bei denen ich eine acute gonorrhoische Endometritis corporis uteri im Frühwochenbett constatirt hatte, zur Nachnntersuchung wieder zu bestellen, um in erster Linie die Ansichten Noeggenrath's und Bumm's über die sogenannte secundäre oder Einkindsterilität zu prüfen.

Während nach Bumm die Gonorrhoe, so lange sie auf den Cervikalcanal beschränkt ist, die Fortpflanzung der Frau nicht ausschliesst, spielt dagegen die Gonorrhoe im Wochenbett eine viel grössere Rolle, weil im Puerperium die Infection so häufig auf die Corpushöhle und die Tuben übergreift, so dass jede weitere Conception, wenn das Beckenbauchfell verändert ist, abgeschnitten ist.

Von den Fällen, bei denen ich eine gonorrhoische Infection der Corpushöhle im Frühwochenbett feststellen konnte, haben sich im Ganzen. 36 Fälle zur Nachuntersuchung gestellt oder es konnte wenigstens von ihnen brieflich Nachricht erhalten werden. Die Dauer der Beobachtungszeit umfasst ca. 5 Jahre.

Von diesen 36 Fällen sind im Verlauf dieser 5 Jahre der Nachbeobachtung trotz häufiger Conceptionsmöglichkeit 13 steril geblieben, also über $1 / 3$ derselben; bei einer weiteren ist im nächsten Jahre nach der Frühwochenbettsinfection noch ein Abort in der 6. Woche eingetreten, wiederum mit schwerem Fieber post abortum verbunden, seit dieser Zeit ist auch hier ebenfalls keine Schwangerschaft mehr erfolgt. Im Fall No. 12 unserer Beobachtungen bestand die Conceptionsmöglichkeit erst seit einem Jahre; in dieser Zeit ist ebenfalls keine Schwängerung eingetreten.

In den übrigen 21 Fällen ist Conception erfolgt, und weiter ist bei allen wenigstens einmal die rechtzeitige Geburt eingetreten. Es muss aber bei diesen Frauen, welche concipirt haben, noch besonders hervorgehoben werden, dass in einigen Fällen die Conception trotz häufiger Cohabitationen, welche bald post partum wieder aufgenommen wurden, auffallend spät eintrat, z. B. in den Fällen 16, 33 und 35 erst 5 Jahre nach der überstandenen Wochenbettsintection und in zwei Fällen erst 4 Jahre nach derselben. Bei den übrigen Frauen ist die Schwängerung schon bald nach der gonorrhoischen Frühwochenbettsinfection wieder erfolgt, so in ver- 
schiedenen Fällen schon ein Jahr später; zum Theil ist bei diesen Frauen sogar eine grosse Fruchtbarkeit zu eonstatiren, da mehrere in dem kurzen Zeitintervall von 5 Jahren bis zu drei Ẹtbindungen durchgemacht haben.

Bei verschiedenen Franen konnte für die bestehende Sterilität eine directe Ursache festgestellt werden. So sind in den Fällen No. 24 und 30 schwere Störungen nach der Wochenbettsinfection eingetreten, sodass wegen gonorrhoischer Pyosalpinx die Exstirpation der Tubensäcke ausgeführt wurde; ausserdem konnten entzündliche Adnextumoren bezw. Verwachsungen des Uterus und der Adnexe in mehreren Fällen constatirt werden; so bestanden im Fall 14 und 27 bei der Wiedervorstellung 5 Jahre post partum deutliche Sactosalpingen neben dem Uterus, und in zwei weiteren Fällen (17 u. 22) war der Uterus bei der Nachuntersuchung hinten breit fixirt.

Besonders erwähnenswerth erscheint es mir, dass manchmal trotz anfänglich nachgewiesenen Ergriffenseins: des Beckenbauchfells dennoch später Schwängerung eintreten kann.

Bei der schweren gonorrhoischen Frühwochenbetterkrankung in Fall No. 8 war ein gonorrhoisches pelviperitonitisches Exsudat im Douglas durch Punction und durch bakteriologische Untersuchung féstgestellt worden. Hier war zunächst trotz häufiger Cohabitationen voljständige Sterilität; 4 Jahre nach der gonorrhoischen Wochenbetterkrankung trat aber Conception ein: Nachdem einmal der Process jetzt zur Ausheilung gekommen war, erfolgte jedes Jahr Schwängerung, ein Zeichen dafür, dass mit grösster Wahrscheinlichkeit die vorhergehende temporäre Sterilität der Frau nicht durch den Mann bedingt gewesen war. Schliesslich erfolgte bei dieser Frau noch nachträglich eine Tubenschwangerschaft, und zwar eine Tubenruptur im zweiten Monat. Tch möchte dieses Ereigniss mehr für einen Zufall erklären, als etwa durch noch bestehende Verwachsungen und Unwegsamkeit der Tuben bedingt.

In verschiedenen Fällen haben wir bei den der gonorrhoischen Wochenbettsinfection folgenden Geburten sehr schön die zuerst von Noeggerath gemachte Beobachtung bestätigen können, dass wiederum Fieber im Frühwochenbett eintritt. Es ist hier besonders charakteristisch der Fall 10, bei welchem im Anschluss an die nächsten drei ganz normal verlaufenden Geburten jedesmal wieder Fieber in Wochenbett einsetzte; ebenso der Fall 20, welcher 
trotz normaler- Entbindungen bei den drei nächsten Wochenbetten stets lange fieberte, nach der ersten Geburt sogar 14 Wochen. Gewiss ist aus der Anamnese nicht mit Sicherheit zu entnehmen, dass die folgenden Wochenbetten wiederum durch Ascendiren der bestehenden Cervixgonorrhoe in die Corpushöhle gestört sind, doch ist es immerhin nach den vorausgehenden Beobachtungen anderer Autoren sehr wahrscheinlich gemacht.

Die Bedeutung, welche die gonorrhoische Wochenbettserkrankung für die "Sterilität der Frau hat, tritt noch deutlicher hervor, wenn wir sie vergleichen mit anderen Wochenbettsinfectionen, z. B. mit der acuten Infection durch. Fäulnissbacterien oder den Streptococeus pyogenes puerperalis. Da ich während der Untersuchung über die Wochenbettsgonorrhoe gleichzeitig Untersuchungen angestellt hatte über die Infection der Uterushöhle im Wochenbett mit -dem Streptococcus pyogenes und den Fäulnisnbacterien, so wurden auch diese Frauen wiederbestellt und nachuntersucht, um über deren weiteres Schicksal orientirt zu sein, vor allem, um über die Häufigkeit der Conception bei diesen Frauen im Vergleich zu den gonorrhoisch erkrankten Frauen etwas zu erfahren. Leider ist es uns bisher nicht gelangen, sämmtliche Frauen wieder aufzufinden, sondern wir haben nur über einen gewissen Procentsatz derselben etwas in Erfahrung bringen und dieselben untersuchen können. Die Beobachtungen, welche wir hierbei gemacht haben, werden ausführlich in einer demnächst erscheinenden Dissertation von Lampe mitgetheilt werden. Ich hebe an dieser Stelle nur dasjenige hervor, was für die vergleichende Beurtheilung mir von Wichtigkeit erscheint.

Wenn wir zunächst diejenigen Fälle, bei denen eine Infection mit Fäulnissbacterien im Frühwochenbett constatirt werden konnte, zum Vergleich heranziehen, so wurden 30 Fälle eine längere Zeit (ca. 5 Jahre) nach der Infection beobachtet.

Von diesen haben 5 trotz reichlicher Cohabitationen nach der Geburt keine Schwangerschaft wieder durchgemacht. Hiervon kommen aber 3 sofort in Wegfall, weil eine Wöchnerin gleich nach der Entbindung eine frische Gonorrhoe acquirirte und ein Jahr später von Saenger wegen doppelseitiger gonorrhoischer Pyosalpinx operirt wurde. Saenger führte die Salpingooophorectomie aus; laut Protokoll, welches uns von Saenger in liebenswürdigster Weise zur Verfügung gestellt wurde, konnte mit Sicherheit festgestellt werden, dass es sich hier um eine gonorrhoische Saktosalpinx handelte, 
412 Kroenig, Zur Prognose der ascendirten Gonorrhoe beim. Weibe.

weil gleichzeitig bei der Frau eine gonorrhoische Uethritis, Bartholinitis und Vulvitis mit dem mikroskopischen Nachweis von Gonokokken bestand.

Bei den anderen zwei Fällen handelt es sich in einem Falle um eine Puella publica, welche bald nach der Entbindung in einem hiesigen Bordell internirt wurde, und z. Zt. sich daselbst auch noch befindet, sodass also auch hier eine nachträgliche gonorrhoische Infection mit Sicherheit anzunehmen ist. In dem letzten Falle hatte der Mann eine sogenannte Wochenbettgonorrhoe acquirirt, indem er den Aufenthalt seiner Frau in der Klinik dazu benutzt hatte, um sich anderwärts frisch mit einem acuten Tripper zu inficiren. Die Frau erkrankte sehr bald nach der Wiederaufnahme des Geschlechtsverkehrs an starkem, eitrigen Ausfluss aus der Scheide, Brennen beim Wasserlassen u. s. w., sodass auch hier mit grösster Wahrscheinlichkeit eine nachträglich erfolgte gonorrhoische Infection vorliegt. Für die bestehende Sterilität in diesen 3 Fällen kann daher nicht die Infection mit Fäulnissbakterien im Frühwochenbett verantwortlich gemacht werden, sondern z. Th. mit Sicherheit, z. Th. mit grösster Wahrscheinlichkeit die nachfolgende gonorrhoische Infection.

Infolge dessen ist trotz reichlicher Cohabitationen nur in zwei Fällen nach Fäulnissinfection im Wochenbett keine Schwängerung eingetreten; in allen anderen Fällen, in welchen Conceptionsmöglichkeit bestand, sind auch Geburten erfolgt und zwar in einem Falle bis $z u$ vier in der fünfjährigen Beobachtungszeit, bei vielen Wöchnerinnen drei; manche allerdings haben nur eine Geburt in der Zeit nach der Wochenbettinfection noch durchgemacht.

Wenn auch die Zahlen klein sind, so ist doch immerhin ein so auffallender Untersehied zwischen dem Verhalten nach der Infection der Corpushöhle mit Gonokokken und der Infection mit Fäulnissbakterien in Bezug auf nachfolgende Sterilität, dass wir wohl nicht fehl gehen, wenn wir in obigen Fällen der Gonorrhoe als solcher, auch dann, wenn nachträglich keine Tubenerkrankung festgeslellt werden konnte, die Schuld an der Sterilität des Weibes zusehreiben.

Wie steht es mit der Streptokokkeninfection im Frühwochenbett? Nachuntersucht konnten bisher 46 Fälle werden.

Bei 10 Frauen erfolgte in der fünfjährigen Beobachtungszeit keine weitere Schwängerung, wobei allerdings erwähnt werden muss, dass in zwei Fällen auch nur ganz vorübergehender Geschlechtsverkehr 
Kroenig, Zur Prognose der ascendirten Gonorrhoe beim. Weibe. 413

bestand. In den übrigen acht Fällen war trotz zahlreicher Cohabitationen keine Schwängerung eingetreten.

Von diesen ist ein Fall einige Wochen nach der Entbindung in hiesiger Klinik wegen fixirter Retroflexio operirt worden; es wurde die Ventrifixura uteri gemacht und dabei festgestellt, dass die Tuben beiderseits verdickt und der Pavillon der Tube beiderseits verschlossen war. Bei einer zweiten Wöchnerin ist ebenfalls bald nach der Entbindung eine Operation ausgeführt, ohne dass wir über die Art derselben etwas eruiren konnten.

In einem Fall hatte schon wäbrend der klinischen Behandlung bald nach der Geburt die Streptokokkeninfection klinisch nachweisbar den primären Infectionsherd, die Corpushöhle, überschritten; es hatte sich wegen eines Streptokokkenempyems die Thoracocenthese und Entleerung der Eiters nothwendig gemacht.

In den übrigen 5 Fällen lässt sich keine besondere Ursache für die Sterilität feststellen, vor Allem nicht aus der Anamnese eine nachträglich acquirirte Gonorrhoe entnehmen, sodass wir vielleicht diese Fälle auf Rechnung der Streptokokkeninfection setzen dürfen.

In den übrigen 34 Fällen, in welchen die Conceptionsmögliehkeit gegeben war, ist auch Conception erfolgt, und zwar haben auch hier wiederum viele Frauen eine grosse Fruchtbarkeit gezeigt, indem nicht weniger als 9 Frauen in der kurzen Beobachtungszeit von 5 Jahren drei und vier Kinder, viele Frauen zwei, vereinzelte nur ein Kind gebaren.

Wenn wir daher auch bei der Streptokokkeninfection des puerperalen Endometriums häufiger ein Nichteintreten der Conception in den nächsten 5-6 Jahren beobachten, als bei der Infection mit den Fäulnissbacterien, so ist doch im Vergleich mit der gonorrhoischen Wochenbettinfection der Einfluss der Infection mit dem Streptococcus pyogenes auf die Sterilität der Frau ein viel geringerer.

Wir können daher dem Ausspruch Bumm's beistimmen, dass das Ergriffenwerden der Corpushöhle im Frühwochenbett mit Gonokokken eine ungünstige Prognose für die weitere Empfängniss der Frau abgiebt; doch dürfen wir auch hier die Prognose nicht so ungünstig wie Nöggerath und Glünder stellen.

Aus unseren Betrachtungen ergiebt sich für die Therapie der ascendirten Gonorrhoe, wenn ich dieselbe mit ganz wenigen Worten streifen darf, Folgendes. Acute, selbst heftige peritonitische Er- 
414 Kroenig; Zur Prognose der ascendirten Gonorrhoe beim Weibe.

scheinungen bei gonorrhoischen Adnexerkrankungen sollen uns nicht zu einem operativen Vorgehen verleiten; wir müssen uns der durch die Erfahrung gegebenen Thatsachen bewusst bleiben, dass selbst bei Ausbildung grosser Pyosalpinxsäcke oft nach kurzdauernder Behandlung eine so schnelle Besserung der Beschwerden eintritt, dass die Frauen ihre Arbeit wieder aufnehmen können. Wenn auch erneute recidivirende. Schmerzattacken eintreten, so sind dieșelben im Allgemeinen in ihrer Heftigkeit gegenüber dem ersten Anfall wesentlich geringer, so dass wir anch im Hinblick auf eventuelle Recidive uns nicht gleich zur Operation entschliessen dürfen.

Unsere Zusammenstellung der principiell conservativ behandelten Fälle hat aber doch ergeben, dass in manchen, wenn auch vereinzelten Fällen wegen der langanhaltenden Beschwerden die Operation erwünscht sein kann. Hierbei muss aber berücksichtigt werden, dass bei der heutigen Technik dureh die Operation allein den Frauen eine definitive Beschwerdefreiheit nicht garantirt werden kann.

Die besten Erfolge scheint die Exstirpation der gesammten Genitalien zu haben, wenn nicht die Beschwerden, welche die Castration als solche mit sich bringt, zu grosse Belästigungen der Frau herbeiführen. Bei Frauen nahe der Klimax, bei welchen nachweislich in Folge der künstlich antecipirten Klimax keine besonderen Beschwerden entstehen, empfiehlt es sich daher, wenn die Beschwerden in Folge der gonorrhoischen Adnexerkrankungen andauernd sind, zur Exstirpation des Uterus, der Tuben und beider Ovarien zu schreiten. Bei den Frauen auf der Höhe der Geschlechtsreife ist dagegen die Castration zu unterlassen, und die weniger radicalen Verfabren, selbst wenn sie nicht so günstige Dauererfolge erzielen, zu empfehlen. Diese können bestehen in Entfernung der Tubensäcke bezw. der vaginalen Eröffnung von Serocelen, pelviperitonitischen Exsudaten, auch in Eröffnung von eitergefüllten Tubensäcken, wie sie in allerletzter Zeit als conservirende. Operationsmethoden in der Leipziger Klinik gemacht werden.

In über 90 pCt. der Fälle ist aber, selbst bei Frauen der arbeitenden Klasse die Prognose der rein exspectativen Methode eine so günstige, dass eine operative Maassnahme überhaupt nicht nothwendig ist.

Nur eine Frage bedarf noch kurz der Erörterung, weil ohne diese die Besprechung, der Prognose der asçendirten Gonorrhoe un- 
Karoenig; Zur Prognose der ascendirten Gonorrhoe beim Weibe. 415

vollkommen wäre, nämlich ob nicht durch längeres Abwarten bei sehr acuten stürmischen, peritonitischen Erscheinungen ein tödtlicher Ausgang durch die Gonorrhoe erfolgen kann. Unter den 74 oben erwähnten Fällen von ascendirter Gonorrhoe ist kein Exitus erfolgt, doch ist eine so kleine Zahl natürlich hierin nicht massgebend, sondern es müssen die Erfahrungen auch anderer Autoren mit herangezogen werden.

Jedem, der Gelegenheit gehabt hat; eine Patientin mit gonorrhoischer Pyosalpinx in hochacutem Stadium zu sehen, oder dann, wenn ein Pyosalpinxsack durch plötzliche Gewalteinwirkung geplatzt ist und Eiter sich auf das Beckenbauchfell ergossen hat, wird die Schw ere der Krankheitserscheinung sicherlich unvergesslich bleiben. Wir wissen heute durch die Untersuchungen von Wertheim, Menge, Döderlein, Bröse und vielen anderen, dass hierbei eine allgemeine Peritonitis entstehen kann. Während meiner Assistentenzeit hatte ich selbst Gelegenheit einen derartigen Fall von gonorrhoischer Peritonitis zu beobachten. Es ist diese Krankengeschichte ausführlich ron Menge (Centr. f. Gynaek. 1893, p. 457) wiedergegeben. Der in die Bauchhöhle ergossene bei der Operation nachgewiesene Eiter hatte eine allgemeine, serös-eitrige Peritonitis hervorgerufen.

Bei der Besprechung dieses Falles wirft Menge schon die Frage auf, ob hier wirklieh eine Indicatio vitalis zum operativen Eingriff vorgelegen hat, und kommt zu dem Resultat, dass man doch die Operation wenigstens noch hätte hinausschieben können.

Bröse ${ }^{1}$ ) berichtet i. J. 1896 über einen ähnlichen Fall, bei welchem ebenfalls nach Lösung des fixirten retroflectirten Uterus in Narkose eine allgemeine Peritonitis eintrat. Bröse verwarf die von ärztlicher Seite vorgeschlagene.Laparotomie und verhielt sich trotz des anscheinend sehr besorgnisserregenden Zustandes der Patientin exspectativ mit dem Erfolg, dass die Patientin in verhältnissmässig kurzer Zeit genas. Ebenso verfuhr Bröse wiederum mit günstigem Ausgang in einem zweiten Falle.

Durchbrüche der gonorrhoischen Saktosalpinx in Nachbarorgane, vor Allem in den Darm, kommen sicherlich vor; aber auch sie nehmen gewöhnlich noch einen günstigen Verlauf.

Wir dürfen daher wohl zur Zeit mit Bumm annehmen, dass

1) Broese, Ueber die diffuse gonorrhoische Peritonitis. Berliner klinische Wochenschrift. 1896. No. 35. 
416 Kroenig, Zur Prognose der ascendirten Gonorrhoe beim Weibe.

der gonorrhoische Process niemals eine directe Gefährdung des Lebens bedingt.

\section{Klinischer Verlauf bei Gonorrhoe der Tuben und des Beckenbauchfells:}

Fall 1. S., Edwine, Dachdeckersfrau, 21 Jahre alt. Klinische Behandlung vom 20. 2. bis 6. 3. 1897.

Diagnose: Pyosalpinx duplex gonorrhoica.

Therapie: wie oben angegeben.

Anamnese: Patientin machte 1895 eine normale Entbindung durch mit ungestörtem Wochenbett. Beschwerden bestehen erst seit 14 Tagen und zwar seit der letzten zur rechten Zeit erschienenen Periode; seit dieser Zeit besteht starke Blutung verbunden mit wehenartigen Schraerzen, die bis zum Tage der Aufnabme anhalten. Die Schmerzen sind besonders heftig bei Stuhlverstopfung.

Status: Abdomen druckempfindlich. Ausführungsgänge der Bartholin'schen Drüsen geröthet, ebenso die Schleimbaut der Urethra. Uterus anteflectirt, nicht druckempfindlich. Rechts vom Uterus ist ein circa emueigrosser, druckempfindlicher Tumor von ziemlich derber Consistenz, welcher auf der Unterlage verwachsen und gegen die Umgebung nur undeutlich abgrenzbar ist; er zeigt undeutliche Fluctuation. Die rechte Tube ist am uterinen Ende gleich verdickt zu fühlen. Linke Tube ebenfalls verdickt und mit dem wenig vergrösserten Ovarium in ein Convolut von Tumoren von etwa insgesammt Gänseeigrösse verwandelt. Sowohl im Urethral-, als auch im Cervicalsecret sind Gonococcen nachweisbar.

Patientin verlässt am 6. 3. 1897 die Anstalt. Die Schmerzen haben nachgelassen, und die Tumoren sind bedentend kleiner geworden.

Befund im Mai 1899: Patientin hat nie wieder Beschwerden gehabt, sondern bald nach der Entlassung aus der Klinik ohne Beschwerden arbeiten können; sie fühlt sich ganz wohl. Conception ist trotz häufiger Cohabitationen nicht eingetreten.

Status: Uterus retrovertirt, flectiri, lässt sich nicht aufrichten, von normaler Grösse und Consistenz. Die beiden Tuben und Ovarien sind nicht mehr verdickt oder vergrössert, aber beide mit der Umgebung adbärent.

Fall 2. B., Martha, Zimmermannsfrau, 28 Jahre alt. Klinische Behandlung 20. 4. bis 15. 5. 1897.

Diagnose: Pyosalpinx gonorrhoïca praecipue sinistra.

Anamnese: Periode stit dem 18. Lebensjahre alle 3 Wochen regelmässig 8 Tage lang andauernd, stark, aber ohne Schmerzen. Zwei normale Geburten 1893 und 1894. Seit der letzten Geburt bestehen siarke, ziehende, stechende Schmerzen in der linken Unterleibsseite mit Kreuzschmerzen verbunden; seit dem 30. 3. 1897 ist Patientin bettlägerig. Nach der Geburt trat ein eiterig schleimiger Ausfluss aus den Genitalien ein mit starkem Brennen beim Wasserlassen; seit dieser Zeit ist auch die Periode unregelmässig geworden und sehr stark.

Status: Uterus dextro- und retroponirt, anteflectirt, schwer beweglich. Die linke Tube, am uterinen Ende verdickt, ist mit dem linken Oxarium, welches nur wenig vergrössert erscheint, fest auf dem Becken- 
boden fixirt. Rechte Tube und rechtes Ovarium zeigen ungefähr gleichen Befund; auch hier keine eigentliche Sackbildung.

Am 15. 5. 1897 wird Patientin gebessert in poliklinische Behandlung entlassen.

Bei der Vorstellung Mai 1899 gab sie Folgendes an: Nach der Entlassung aus der Klinik wurde sie noch poliklinisch mit Scheidentampons und Scheidenspülungen behandelt. Hierdurch trat zunächst Besserung ein, die aber nicht lange anhielt. Das Leiden verschlimmerte sich in der Folgezeit wiederum; Patientin bekam starke Schmerzen in beiden Unterleibsseiten und im Kreuz mit Drängen nach unten. Sie musste 6 Wochen das Bett hüten. und war wiederum in ärztlicher Behandlung; es wurden ihr Sitzbäder und Ausspülungen rerordnet. Dadurch trat wiederum zeitweise Besserung ein, aber Pat. musste häufig von Neuem ärztliche Hilfe in Anspruch nehmen. Die Periode blieb unregelmässig, alle 4 bis 6 Wochen eintretend, 8 Tage lang, mit starkem Blutabgang; vor und während der Periode bestehen ziehende, wehenartige Schmerzen. Anfang des Jahres 1899 wurde sie in Berlin in der Poliklinik der Universitäts-Frauenklinik 6 Wochen lang behandelt. Seit der Zeit geht es ihr wesentlich besser, vor allem haben die Schmerzen fast vollständig aufgebört. Einigermaassen anstrengende Hausarbeit kann sie auch heute noch nicht verrichten, sondern empfindet hierbei sogleich wieder Beschwerden. Patientin leidet häufig an Kopfschmerzen und fühlt sich im Allgemeinen durch die lange Krankheit sehr angegriffen. Es bestehen Schmerzen bei der Cohabitation. Weder eine Geburt noch eine Fehlgeburt ist trotz zahlreicher Cohabitationen eingetreten.

Nachuntersuchung leider verweigert.

Fall 3. G., Lisette, Kaufmannsfrau, 30 Jahre alt. Klinisch behandelt 17. 6. bis 28. 6. 1897.

Diagnose: Pyosalpinx dextra; Salpingooophoritis sinistra.

Anamnese: Seit einer Entbindung i. J. 1891 besteht bei Patientin etwas weisser Ausfluss, doch zun̈̈chst ohne Schmerzen. Im Juni 1895 traten ziehende Schmerzen im Unterleib ein mit starkem Brennen beim Wasserlassen. Patientin war damals 5 Wochen bettlägerig, hatte einige Tage Fieber und starke Schmerzen beim Stuhlgang und beim Abgang von Blähungen. Aus der Scheide ging stark eitriger, gelblicher, ätzender Fluor ab. Durch Behandlung mit Eisblase und Umschlägen besserten sich die Beschwerden. Seit der Zeit war die Periode unreselmässig, zeitweilig sebr stark und mit längerem Bhatverlust verbunden. Von Ende Mai 1896 bis Nitte Juli 1897 ununterbrochene Blutung, wenn auch nicht sehr stark. Allmälig wurde die Menstruation wieder regelmässig. Erst am 9.6.1897 stellten sich wiederum starke Menorrhagien ein, die 10 Tage lang anhielten.

Am 12. 6. 1897 traten plötzlich sehr heftige Unterleibsschmerzen ein mit starkem Drängen uach unten. Die Schmerzen lokalisirten sich besonders in der rechten Seite und im Kreuz, und waren besonders heftig bei etwas angehaltenem Stuhlgang. Fieber trat nicht auf. Die krampfartigen Schmerzen wiederholten sich, ebenso der Blutabgang, sodass Patientin klinische Behandlung aufsuchte.

Status: Uterus von normaler Grösse, anteflectirt, wenig beweglich. Die linken Adnexe mässig schmerzhaft. Tube am uterinen Ende leicht verdickt. Rechte Tube ebenfalls am uterinen Ende verdickt, bildet mit dem Ovarium einen apfelgrossen, derben Tumor, welcher auf dem Beckenboden fixirt ist und den Douglas nach unten zu verwölbt. 
418 Kroenig, Zur Prognose der ascendirten Gonorrhoe beim Weibe.

Entlassung am 28.6. 1897. Subjectives Befinden besser, die Schmerzen haben wesentlich nachgelassen. Objectiv derselbe Befund zu erheben.

Nachuntersuchung im Mai 1899. Nach der Entlassung aus der Klinik hat Patientin noch 6 Wochen lang sich zu Hause geschont und täglich Sitzbäder genommen. Danach besserten sich die Beschwerden. Nur Ostern 1898 trat noch einmal ein krampfartiger Schmerzanfall im Unterleib ein, welcher sie für einige Zeit ans Bett fesselte, doch gingen die Schmerzen bald wieder vorüber. Zur Zeit füblt sich Patientin sehr wohl und ist voll arbeitsfähig.

Status: Im Allgemeinen derselbe Status wie im Jahre 1897.

Fall 4. W., Bertba, Fabrikarbeiterin, 27 Jabre alt. Klinisch behandelt vom 19. 7. bis 26. 7. 1897.

Diagnose: Pyosalpinx gon. sin. Perisalpingitis dextra.

Patientin erkrankte am 6. 7. d. J. mit stechendem Schmerz in der rechten Seite; sie hatte schon früher bei der letzten Schwangerschaft starken Ausfluss und Brennen beim Wasserlassen gehabt. Die Beschwerden wurden in den letzten Tagen so heftig, dass Patientin am 10. 7. die Arbeit aufgeben musste. Pat. wurde zunächst in der Poliklinik behandelt, dann aber der Klinik überwiesen.

Die Untersuchung ergiebt: die Schleimhaut des Scheideneingangs ohne entzündliche Erscheinungen. Uterus antevertirt, nicht vergrössert. Links vom Uterus, diesem direct anliegend, ein gänseeigrosser, fluctuirender Tumor, aus welchem sich Tube und Ovarium nicht isolirt austasten lassen. Die rechte Tube beginnt am uterinen Ende gleich strangartig verdickt. Das rechte Ovarium ist etwas vergrössert, auf dem Beckenboden adhärent und gegen die Tube nicht sicher abgrenzbar.

Bei der Vorstellung im Mai 1899. gab Patientin an, dass sie nach der Entlassung aus der Klinik zunächst noch eine kurze Zeit sich in poliklinischer Behandlung befunden hätte; sie hat noch 5 Wochen lang Scheidenspülungen, warme Sitzbäder und Priessnitzumschläge um den Leib gemacht. Die Schmerzen haben damals ganz aufgehört. Im Januar 1898 traten aber erneute Schmerzen im Unterleib und Kreuz ein, sodass sie sich wiederum in ärztliche Behandlung begeben musste; schon nach achttägiger Behandlung trat wieder vollständige Besserung ein, Patientin konnte ihre Arbeit wieder ganz aufnehmen. Zur Zeit kommt sie ihrer Beschäftiguug als Waschmädchen ohne Beschwerden nach. Die Periode ist noch unregelmässig alle 2 bis 3 Wochen und fast 8 Tage lang. Kreuz- und Unterleibsschmerzen sind nur vorübergehend vor Eintritt der Periode. Ausfluss besteht nicht mehr. Bei. der Cohabitation geringe Schmerzen. Schwanger ist sie trotz häufigen Coïtus und trotzdem sie keine Vorsichtsmassregeln gebraucht nicht wieder geworden.

Status: Uterus anteflectirt, beweglich. Beide Tuben etwas verdickt und auf Druck empfindlich. Die Ovarien sind wegen der Schmerzhaftigkeit nicht sicher abtastbar.

Fall 5. F., Lina, Lageristin, 23 Jahre alt. Klinische Behandlung vom 10. 7. bis 12. 7. 1897.

Diagnose: Pyosalpinx duplex gon.

Anamnese: Stärkere Beschwerden bestehen erst seit kurzer Zeit und zwar seit der letzten Periode. Diese ist auffallend stark gewesen mit stückweise abgehenden Blutmengen. Schmerzen im Leibe treten vorübergehend auf. Seit 2 Jahren wird Patientin vom Arzt wegen geringer Unterleibsschmerzen und Gebärmutterknickung behandelt. 
Status: Uterus retrovertirt, flectirt, hinten fixirt, nicht aufzurichten. Beiderseits vom Uterus sind gänseeigrosse, unverschiebliche Tumoren zu fühlen, aus denen sich Tube und Ovarium nicht isolirt austasten lassen.

Bei der Vorstellung im Juni 1899 giebt Patientin an, dass sie nach ihrer Entlassung aus der Klinik zunächst noch dieselben Schmerzen im Unterleibe gehabt hat, zumal ihre Beschäftigung als Lageristin nur wenig Ruhe erlaubte. Besonders wenn sie schwer heben musste, traten krampfartige Schmerzen im Unterleib auf. Die Periode ist regelmässig alle 4 Wochen, nur etwas sehr stark und von langer Dauer. Seit vorigem Jahre ist Patientin wegen Bleichsucbt und Lungenkatarrh in ärztlicher Belsandlung und nicht mebr voll arbeitsfähig; auch jetzt treten noch manchmal Schmerzen im Unterleib auf. Schwangerschaft ist trotz häufiger Cohabitation nicht erfolgt.

Status: Uterus retrovertirt, flectirt, hinten fixirt. Beide Tuben am uterinen Ende gleich verdickt. Beide Ovarien im Douglas fixirt, doch nicht vergrössert. Tumoren nicht mehr zu fühlen.

Fall 6. G., Ella, Maschinistenehefrau, 23 Jahre alt. Klinisch behandelt rom 16. 9. bis 8.10 .1897 .

Diagnose: Endometritis cum fluore. Retroversio uteri fixati. Pyosalpinx duplex gon.

Anamnese: Seit der letzten Entbindung im Januar 1896 besteht starker eitriger Ausfluss und zeitweise Schmerzanfälle, besonders während der Regel. Ausserdem tritt die früher regelmässige Periode unregelmässig auf; zum Theil heftige Metrorrhagien. Seit 8 Tagen ist der Schmerz im Unterleib so stark, dass Patientin die Klinik aufsucht.

Status: Uterus retrovertirt, hinten breit fixirt, von normaler Grösse. Beiderseits sind Tuben und Ovarien in gänseeigrosse, druckempfindliche Tịmoren verwandelt, welche auf dem Beckenboden fest adhärent sind. Bei der Aufnahme $39,6^{\circ}$; Puls sehr frequent. Nach 2 Tagen fällt die Temperatur zur Norm $a b$ und bleibt normal. Bei der Entlassung ist der objective Befund noch derselbe; doch sind die Beschwerden wesentlich gebessert.

Bei ibrer Vorstellung im Mai 1899 giebt Patientin an, dass sie nach der Entlassung aus der Klinik noch einige Zeit in poliklinischer Behandlung gewesen ist und dort mit Sitzbädern, Scheideneinlagen und innerlich mit Pulvern gegen die Gebärmutterblutungen behandelt ist. Die Periode blieb die nächste Zeit unregelmässig; trat alle 2-3 Wochen auf und dauerte 8 Tage lang mit starkem Blutverlust. Einige Tage vor und nach der Periode bestanden Schmerzen im Unterleib; doch sind wirkliche Schmerzattacken nie wieder aufgetreten. Zur Zeit besteht noch Ausfluss, weswegen Patientin Scheidenspülungen macht.

Das Allgemeinbefinden der Patientin ist zur Zeit gut; sie ist voll arbeitsfähig, ohne dass ihr selbst schwere körperliche Ärbeit Schmerzen verursacht. Trotz häufiger Cohabitationen ist Patientin nicht wieder schwanger geworden.

Status: Uterus hinten fixirt. Beiderseits neben dem Uterus befinden sich noch gänseeigrasse, auf Druck empfindliche Tumoren, aus denen sich Tuben und Ovarium nicht isolirt austasten lassen. Die Tumoren sind an der hinteren Beckenwand fixirt.

Fall 7. S., Johanne, Gelbgiessersehefrau, 29 Jahre alt. Klinisch behandelt vom 23. 11. bis 2. 12. 1897. 
Diagnose: Bartholinit. gon., Endometritis cervicis gon.; Pyosalpinx duplex gon.

Patientin war früher gesund, hat 3 normale Geburten und. Wochenbetten durchgemacht, und eine Frühgeburt im Jalıre 1896. Ihre Beschwerden rühren von der letzten Fehlgeburt her, und zwar bestehen dieselben in einem stark gelblich gefärbtem Ausfluss, za welchem sich in der letzten Zeit äusserst beftiges Brenuen beim Wasserlassen gesellte. Seit Anfang November d. J. sind heftige Unterleibsschmerzen aufgetreten, welche besonders Nachts exacerbiren und ihr den Schlaf rauben.

Status: Aus der Urethra lässt sich ein gelblich eitriges Secret ausdrücken, in welchem zahlreiche Gonococcen gefunden werden; ebenso sind Gonokokken im Cervixsecret und im Seeret der Bartholin'schen Drüsen. Der Uterus ist nicht vergrössert, antevertirt, schwer beweglich. Die linke Tube ist am uterinen Ende gleich verdickt und geht nach links und hinten über in einen Tumor von etwa Regensburger Wurst-Grösse, aus welchem sich Tube und Ovarien nicht mehr isolirt austasten lassen. Rechts rom Uterus ist ein ebenso grosses Convolut von Tumoren zu fühlen. Beide Geschwülste sind auf dem Beckenboden adhärent und füllen zum Theil den Douglas aus. Am 2. 12. wird Patientin in poliklinische Behandlung entlassen. Die Schmerzen haben sich bedeutend verringert. Der objective Befund ist jedoch noch ungefähr derselbe.

Bei der Vorstellung im Mai 1899 giebt Patientin an, dass sie noch ein Jahr lang eitrigen Ausfluss aus der Scheide und Brennen beim Wasserlassen gehabt hat. Die Periode ist ganz regelmässig geworden und danert mit mässiger Stärke nur 3-4 Tage an. Vor der Periode treten manchmal noch etwas Kreuzschmerzen auf, doch hat Patientin nie wieder Schmerzen im Unterleib gehabt. Sie ist zur Zeit voll arbeitsfähig und fühlt sich ganz wohl. Trotz häufig ausgeführter Cohabitation, welche schmerzlos sind, ist niemals Schwangersehaft wieder eingetreten.

Status im Mai 1899: Uterus antevertirt, mässig beweglich. Die Cervix hinten fixirt. Die Tuben am uterinén Ende isolirt anstastbar, gehen in etwas über taubeneigrosse, seitlich gelegene, druckempfindliche Tumoren über, aus denen das Ovarium beiderseits nicht austastbar ist, und welche auf dem. Beckenboden adhärent sind.

Fall 8. S., Gertrud, Zusehneidersehefrau, 22 Jahre alt.

Klinische Behandlung: 7. 12. bis 12. 12. 1897.

Diagnose: Pyosalpinx dupl. gon.

Anamnese: Im Jahre 1895 ist Patientin mit Zange entbunden; das Kind bekam eine Augenentzündung, welche nach Anwendung von Höllensteinlösung heilte. Im darauffolgenden Jabre machte Patientin eine Fehlgeburt durch. Seit der letzten Periode vom 23. bis 28.11.97 sind die seit mehreren Monaten bestehenden Leib- und Kreuzschmerzen besonders heftig, sodass Patientin die Klinik aufsucht.

Status: Am Scheideneingang sind keine entzündlichen Veränderungen. Der Uterus ist anteflectirt, schwer beweglich. Beiderseits rom Uterus, direkt demselben anliegend, ist ein Convolut von Tumoren zu fühlen von etwa emueigrösse, aus welchen sich Tube und Ovarium nicht isolirt austasten lassen. Die Tube ist am uterinen Ende beiderseits gleich verdickt.

Am 12. 12. wird Patientin, ohne dass Besserung eingetreten ist, aus der Klinik entlassen. 
Patientin war nicht zu bewegen, zur Nachuntersuchung wieder in die Klinik zu kommen. Der Ehemamn der Patientin theilte aber auf die Anfrage im Mai 1899 mit, dass seine Frau nach der Entlassung ans der Klinik noch 14 Tage lang das Bett gehütet und während dieser Zeit fortwährend kühle Umschläge gemacht hätto; danach habe sie noch eine Zeit lang täglich Sitzbäder genommen, welche vollständige Gesundheit und Beschwerdenfreiheit herbeigeführt hätten. Schwanger ist sie nicht wieder geworden.

Fall 9. W., Elise. Verkäuferin, 21 Jahre alt.

Klinische Behandlung: 28. 1. bis 10. 2. 1898.

Diagnose: Pyosalpinx dupl. gon.

Anamnese: Im Februar 1897 ist Patientin. wegen stark eitrigen Ausflusses aus der Scheide ärztlich behandelt worden. Im August 1897 stellten sich so heftige Beschwerden im Unterleib bei jeder körperlichen Bewegung ein, dass Patientin sich zu Bett legen musste. Nach siebenwöchiger Bettruhe liessen die Schmerzen bedeutend nach, doch hörten sie niemals voliständig auf, sondern Patientin ist seit August vorigen Jahres arbeitsunfähig.

Status: Ausführungsgänge beider Bartholinscher Drüsen geschwollen und geröthet. Aus der Urethra lässt sich kein Secret ausdrücken. Uterus klein, antevertirt, schwer beweglich. Links rom Uterus ist die Tube am uterinen Ende gleich verdickt zu fühlen, geht dann über in ein Convolut von Tumoren von über Gänseeigrösse, aus welchem sich Tube und Ovarium nicht isolirt austasten lassen. Rechts und nach hinten vom Uterus ist ein ungefähr gleichgrosser Tumor zu fühlen, welcher mit dem Uterus und mit dem Beckenboden fest verwachsen ist. Im Cervixsecret sind Gonococcen nachzuweisen. -

Befund Il ai 1899: Nach der Entlassung aus der Klinik ist Patientin noch eine Zeit lang in ärzticher Behandlung in Berlin gewesen; es wurde dort eine Auskratzung gemacht. Bald danach trat Besserung ein. Patientin hat zur Zeit keine Beschwerden mehr, fühlt sich ganz wohl und ist vollkommen arbeitsfähig. Eine Conception ist trotz reichlicher Cohabitationen nicht eingetreten.

Status: Uterus klein, antevertirt. Linke Tube noch daumendick, auf Druck wenig schmerzhaft; dahinter das Ovarium wallnussgross, ebenfalls kaum druckempfindlich. Rechte Tube am uterinen Ende bleistiftdick, schwillt dann an und ist mit dem wenig vergrösserten Ovarium verwachsen und anf dem Beckenboden adhärent.

Fall 10. H., Elisabeth, Schutzmannsfrau, 26 Jakre alt.

Klinische Behandlung: 27. 2. bis 14. 3. 1896.

Diagnose: Endometritis cervicis gon.; Pyosalpinx dupl. gon.

Anamnese: Patientin leidet seit mehreren Jahren, seit sie mit ihrem Mann verheirathet ist, an anfangs geringem, allmälig immer stärker werdendem gelblichen Ausfluss. Im November 1895 consultirte sie deswegen einen Arzt, welcher Gebärmutterkatarrh und eine Knickung der Gebärmutter feststellte. Ein eingelegter Ring verursachte so heftige Schmerzen, dass er nach 14. Tagen schon wieder entfernt werden musste. Ihren 2 Kindern wurden direkt nach der Geburt auf ärztliche Anordnung die Augen mit Höllensteinlösung behandelt; trotzdem stellte sich bei beiden Kindern nach der Geburt eine Augenentzündung ein, die aber auf Behandlung sich bald besserte. So lange der Ausfluss besteht, leidet Patientin auch an Unterleibschmerzen, welche im Allgemeinen gering sind, aber zeitweise exacerbiren. 
Status: Uterus retroponirt, anteflectirt. Beiderseits vom Uterus ist ein etwa gänseeigrosses Convolut von Tumoren zu füblen, aus welchem sich Tube und Ovarien nicht isolirt austasten lassen. Die Tuben beginnen am uterinen Ende beiderseits gleich deutlich verdickt.

Bei der Vorstellung im Mai 1899 giebt Patientin an, dass sie wegen der Unterleibsschmerzen und des Ausflusses zu Hause noch weiter Sitzbäder und Scheidenspülungen gemacht hat. Danach trat sehr bald völlige Besserung ein. Zur Zeit fühlt sie sich sehr wohl und kann ohne Beschwerden ihre Hausarbeit verrichten. Die Periode ist regelmässig; von normaler Stärke.

Status: Uterus von normaler Grösse, retrovertirt, fixirt, lässt sich nicht aufrichten. Beide Tuben wenig verdickt. Rechtes Ovarium von normaler Grösse, in der Beweglichkeit wenig behindert. Linkes Ovarium ebenfalls von normaler Grösse, aber fest an der hinteren Beckenwand fixirt.

Fall 11. S., Pauline, Fabrikarbeitersehefrau, 30 Jahre alt.

Klinische Behandlung. vom 11. 5. bis 16. 5. 1896.

Diagnose: Pyosalpinx dupl. gon.

Anamnese: 'Patientin hat 5 Entbindungen ohne Kunsthülfe durchgemacht, die letzte Entbindung vor 2 Monaten. Vier Wochen vor der letzten Entbindung trat bei der Patientin während der Schwangerschaft sehr starker Ausfluss ein, welcher gelbgrünliche Flecke in die Wäsche machte. Während der ersten Tage des Wochenbetts bestanden keine Beschwerden; aber bald danach traten Schmerzen auf und zwar besonders ziehende Schmerzen im Unterleib.

Status: Urethralschleimhaut stark geröthet und geschwollen; im Secret Gonococcen. Uterus antevertirt, schwer beweglich. Rechts rom Uterus ist ein etwas über hühnereigrosser Tumor zu fühlen, welcher dem Uterus direkt anliegt, and aus dem sich Tube und Ovarium schwer isolirt austasten lassen. Links vom Uterus ein etwas grösserer Tumor tistbar, welcher auf dem Beckenboden adhärent ist.

Bei der Vorstellung im November 1899 gab Patientin an, dass sie nach der Entlassung aus der Klinik noch eine. Zeit lang wegen ihrer Schmerzen Sitzbäder und Priessnitzumschläge gemacht hat. Bald darauf trat wesentliche Besserung ihrer Schmerzen ein. Ihre Hausarbeit verursacht ihr keine Schmerzen; nur beim Heben schwerer Gegenstände hat Pat. noch manchmal leichtes Ziehen im Unterleib. Schwanger ist sie trotz häufig ausgeführter Cohabitationen nie wieder geworden.

Fall 12. H., Auguste, Friedh ofwächtersehefrau, 31 Jahre alt.

Klinische Behandlung vom 6. 7. bis 18. 7. 1896.

Diagnose: Pyosalpinx dupl. gon.

Anamnese: Patientin hat 12 normale Entbindungen durchgemacht, die letzte Entbindung vor 2 Jahren. Seit langer Zeit klagt Patientin über krampfartige Schmerzen im Leib, welche während der Regel nach dem Rücken hin ausstrahlen. Die Periode ist in letzter Zeit stark gewesen. Vorher heftiges Brennen beim Wasserlassen. Patientin war deswegen in poliklinischer Behandlung. Vor 14 Tagen trat plötzlich ein sehr heftiger Schmerz im Unterleib ein.

Status: Uterus retrovertirt, nicht ganz aufrichtbar, wenig vergrössert. Links vom Uterus ist ein über hübnereigrosser und deutlich fluctuirender Tumor zu fühlen, welcher vom Uterus abgrenzbar und der linken Beckenwand adhärent ist. Die linke Tube am uterinen Ende gleich verdickt, geht in den oben beschriebenen Tumor über, aus dem 
sich das Ovarium nicht isolirt austasten lässt. Rechte Tube ist auf eine längere Strecke hin kleinfingerdick zu fühlen. Rechtes Ovarium nicht vergrössert, auf dem Beckenboden adhärent.

Bei der Vorstellung im Mai 1899 giebt Pat. an, dass sie nach der Entlassung noch häufiger derartige Schmerzattacken gehabt hat. Sobald sie längere Zeit Bettrube hielt, trat stets Besserung ein. Jetzt hat sie noch von Zeit zu Zeit starken Ausfluss, Brennen beim Wasserlassen und häufig starke Schmerzen im Unterleib. Die Periode ist 8 Tage lang, regelmässig, aber ziemlich stark. Pat. meint, dass sie nur wenig seit der Zeit gebessert ist und kann nur leichte Arbeit verrichten. Trotz häufiger Cohabitationen keine Conception.

Status: Uterus antevertirt, beweglich. Beide Tuben etwa kleinfingerdick, auf Druck sehr empfindlich. Die Ovarien wenig vergrössert, auf dem Beckenboden adhärent.

Fall 13. B., Hedwig, Eisendrehersfrau, 34 Jahre alt.

Klinische Behandlung rom 13. 7. bis 24. 7. 1896.

Diagnose: Pyosalpinx dupl. gon.

Anamnese: Pat. hat zwei normale Entbindungen und Wochenbetten durchgemacht, die letzte vor 3 Jahren. Schon vor ihrer Verheirathung hat sie aufallsweise Unterleibsbeschwerden gehabt, sodass sie nicht völlig arbeitsfähig war. Ostern dieses Jahres waren die Beschwerden so stark, dass sie 4 Wochen lang sich ins Bett legen musste, worauf erst altmälig Besserung eintrat. Am 5. 7. 1896 stellten sich erneute, krampfartige Schmerzen, besonders von der linken Seite ausgehend, beim Gehen und Arbeiten ein. Patientin begab sich am 13. 7. in ärztliche Behandlung. Vor längerer Zeit bestand Ausfluss, welcher gelbliche Flecken in die Wäsche machte. Die Periode ist niemals ausgeblieben, doch sind in der letzten Zeit unregelmässige Blutungen aufgetreten, welche zum Theil sehr stark waren.

Status: Uterus anteponirt; links und nach hinten rom Uterus ist ein fast emueigrosser, zum Theil deutlich fluctuirender Tumor zu fühlen, welcher sich von einem weiter rechts gelegenen, gleich grossen. Tumor nur undeutlich abgrenzen lässt. Die Tuben and Ovarien lassen sich neben dem Tumor nicht isolirt austasten.

Bei ihrer Vorstellung im Mai 1899 giebt Pat. an, dass sie nach der Entlassung aus der Klinik ein Jahr lang sich leidlich wohl befunden hat. Im Sommer 1897 traten aber wiederum Beschwerden ein, sodass Pat. wieder in poliklinische Behandlung kam. Sie wurde mit Ichthyoltampons, Sitzbüdern, Scheidenspülungen und vorübergehender Bettruhe behandelt. Darauf trat wesentliche Besserung ein. Kurze Zeit vor ihrer Vorstellung bekam Pat. wiederum Kreuzschmerzen und starken Ausfluss. Die Periode ist alle 3 Wochen $4-5$ Tage lang, mässig stark; nur zeitweise mit Schmerzen verbunden. Trotz Cohabitationen keine Conception. Pat. fühlt sich zur Zeit im Allgemeinen leidlich wohl, ist aber nur zeitweise arbeitsfähig.

Status: Uterus retrofixirt, von normaler Grösse. Beiderseits neben dem Uterus ist ein etwa hühnereigrosser, druckempfindlicher Tumor vou unregelmässiger Form zu fühlen. Die Tuben und Ovarien lassen sich nicht mit Sicherheit isolirt austasten.

Fall 14. L., Victoria, Bremsersfrau, 27 Jabre alt.

Klinische Behandlung vom 29. 5. bis 13. 6. 1895.

Diagnose: Pyosalpinx dupl. gon.

Anamnese: Periode war seit dem 17. Lebensjahre regelmässig 
alle 4 Wochen, 8 Tage lang; in letzter Zeit etwas stärker wie früher. Pat. hat drei normale Entbindungen und Wochenbetten durchgemaeht, die letzte Geburt December 1894:

Nach der letzten Periode, die zur rechten Zeit eintrat, bekam Pat. heftige Schmerzen im Unterleib beiderseits mit Erbrechen. Schon vorher waren zeitweise Schmerzen im Unterleib aufgetreten, welche auf eine Gebärmutterentzündung zurückgeführt wurden, doch jiemals in der Intensität wie jetzt.

Status: Aus der Scheide fliesst reichlich eitriges Secret ab; es lassen sich in 3 Präparaten des Cervixsecrets Gonococcen nicht finden. Uterus ist nicht vergrössert, anteponirt. Links von ihm ist ein wenig schmerzhafter, prall-elastischer Tumor von etwa Strausseneigrösse und glatter Oberfläche zu fühlen. Rechts vom Uterus ist ein gegen die Umgebung undeutlich abgrenzbarer, kleinerer Tumor wie links zu tasten, welcher auf Druck sehr schmerzhatt ist. Tube und Ovarien sind beiderseits nicht isolirt austastbar.

Am 13. 6. 1895 bei der Entlassung aus der Klinik besteht noch ein starker, eitriger Ausfluss aus der Scheide. Die Unterleibsbeschwerden treten nur zeitweise und nicht sehr heftig auf. Der Uterus ist klein, antevertirt. Linke and rechte Tube sind am uterinen Ende verdickt zu fühlen. Die beiderseitigen Tumoren siud bedeutend kleiner geworden, aber auf Druck noch sehr schmerzhaft.

Bei der Vorstellung im Mai 1899 giebt Pat. an, dass sie nach der Entlassung noch Beschwerden gehabt hat, aber niemals mehr von der Intensitat wie ror der Behandlung. Sie hatte weiter Sitzbäder und Priessnitzumschläge um den Leib gemacht.

Im Frühjahx 1897 war sie nochmals in poliklinischer Behandlung wegen erneuter Schmerzen, und war hier mit Scheidentampons und beissen Ausspülungen behandelt, welche schnell Besserung herbeiführten. Die Periode blieb zunüchst noch sehr stark, aber seit einem Jahre sind stärkere Blutungen nicht mehr aufgetreten.

Zur Zeit ist leidliches Wohlbefinden und volle Arbeitsfahigkeit vorhanden. Trotz reichlicher Cobabitationen Sterilität.

Status: Uterus retrovertilt, fixirt; rechte Tube am uterinen Ende schlank, von dem Ovarium, welches nicht vergrössert ist, undeutlich abgrenzbar. Linke Tube schlank; linkes Ovarium nicht vergrössert, hinten fixirt.

Fall 15. R., Bertha, Verkäuferin, 25 Jahre alt.

Klinisehe Behandlung rom 27. 6. bis 6. 7. 1895.

Diagnose: Pyosalpinx dupl. gon.

Anamose: Pat. jst bisher nicht schwanger gewesen. Seit Februar 1895 zuerst geschlechtlicher Verkehr. Seit 5 Wochen bestehen Schmerzen in der linken Unterleibsseite, anfänglich mit Fieber verbunden. Die Beschwerden waren so heftig, dass Patientin arbeitsunfähig war und sich. ins Bett legen musste. Der Arzt machte Einlagen, worauf die stechenden Schmerzen nachliessen und Pat. wieder ins Geschäft gehen konnte. Bald nach Wiederaufnahme der Arbeit traten jedoch die alten Schmerzen wieder auf, sodass sie die KJinik aufsuchte. Vor Beginn der Schmerzen bestand gelblicher, eitriger Ausfluss und heftiges Brennen beim Wasserlassen.

Status: Die Scheidenwände sind rauh. Aus der Scheide fliesst eitriges Secret ab. Die Ausführungsgänge der Bartholinschen Drüsen sind geröthet. Uterus retrovertirt. Linke Tube am uterinen Ende 
gleich verdickt und mit dem linken Ovarium in ein gänseeigrosses Convolut von Tumoren verwandelt. Rechts genau der gleiche Befund wie links. Die Tumoren sind auf dem Beckenboden allseitig adhärent.

Bei der Vorstellung im Mai 1899 giebt Pat. an, dass sie seit 2 Jahren verheirathet ist. Nach der Entlassung aus der Klinik hat sie sich noch 4 Wochen auf dem Lande zur Erbolung anfgehalten. Pat. war dann in der Saenger'schen Poliklinik, wo sie mit Blasenspülungen, heissen Salzwasserbädern und Scheidenspülungen behandelt wurde. Nach dieser Behandlung besserte sich ihr Befinden allmälig; doch traten noch öfter beim Gehen Schmerzen in der linken Seite auf. Die Periode war bis vor 2 Jahren sehr unregelmässig, ist aber jetzt normal, alle 4 Wochen 2 Tage dauernd, schwach und ohne Schmerzen. Zur Zeit fühlt Pat. sich vollständig gesund und ist voll arbeitsfähig; sie hat keinerlei Beschwerden. Trotz häufiger Cohabitationen vor und in der Ehe keine Conception.

Status: Starker, weisser Fluor, etwas Vulvitis. Uterus retrovertirt, fixirt. Beiderseits neben dem Uterus sind reichlich hühnereigrosse, auf Drack nicht empfindliche Tumoren zu fühlen, ais denen sich Tube und Ovarium nicht isolirt austasten lassen.

Fall 16. L., Therese, Steinsetzersehefrau, 24 Jahre alt.

Klinische Behandlung: 24. 9. bis 10. 10. 1894.

Diagnose: Pyosalpinx dupl. gon.

Anamnese: Pat. hat 2 Entbindungen obne Kunsthïlfe durchgemacht. Seit mehreren Wochen bestehen Schmerzen im Unterleib und im Krèz, und manchmal heftige Blutungen ausserhalb der Periode.

Status: Unterleib auf Druck ausserordentlich schmerzhaft, sodass jede bimanuelle Untersuchung unmöglich ist. In Folge dessen wird am 26: 9. eine Narkosenuntersuchung gemacht. Diese ergiebt: Uterus etwas vergrössert, retrovertirt. Rechts und links vom Uterus ist ein etwa faustgrosser, derber Tumor zu fühlen, aus welchem sich Tube und Ovarien nicht austasten lassen. Die Tumoren sind allseitig auf dem Beckenboden und mit dem Uterus adhärent.

Am 10. 10. 1894: Die Schmerzen sind vollständig geschwunden, doch besteht auf Druck noch lebhafte Schmerzempfindlichkeit des Abdomens.

Bei der Vorstellung im Mai 1899 giebt Pat. an, dass sie nach der Entlassung aus der Klinik noch 3 Wochen lang in poliklinischer Behandlung sich befunden habe und mit Ichthyoltampons und Abführmitteln behandelt sei. Darauf völlige Besserung. Einige Zeit danach trat noch einmal eine dreiwöchentliche Blutung auf; seitdem aber ist die Periode regelmässig von normaler Dauer und Stärke, ohne Schmerzen. Auch sonst sind niemals wieder irgend welche Beschwerden aufgetreten. Pat. fühlt sich sehr wohl, ist vollständig arbeitsfähig. Schwanger ist sie seit 1894 trotz häufiger Cohabitationen nicht wieder geworden.

Status: Pat. ist in der letzten Zeit derartig fett geworden, dass wegen Adipositas permagna eine sichere Exploration unmöglich ist.

Fall 17. K., Marie, Arbeitersehefrau, 33 Jahre alt.

Poliklinische Behandlung im August 1897.

Diagnose: Pyosalpinx dupl. gon.

Anamnese: Vor 8 Jahren hat Pat. eine Entbindung mit ärztlicher Hülfe durchgemacht, worauf ein 18 Wochen Ianges Kindbettfieber folgte. Seit 4 Wochen bestehen Schmerzen in der rechten Unterleibs- 
seite, verbunden mit grossem Mattigkeitsgefühl und heftigen Metrorrhagien.

Status: Uterus retrovertirt. Beiderseits neben dem Uterus sind Tumoren von Hühnereigrösse zu fühlen, aus welchen sich Tube und Ovarien nicht isolirt austasten lassen. Die Tumoren sind am Beckenboden adhärent und auf Druck sehr schmerzhaft.

Wegen der starken Metrorrhagien wird zunächst Stypticin gereicht, darauf steht die Blutung ziemlich schnell. Pat. blieb ein ganzes Vierteljahr lang in poliklinischer Behandlung, ohne dass sich ihr Leiden wesentlich besserte. Sie selbst führt es darauf zurück, dass sie sich zu Hause niemals schonen konnte. Sobald sie einen Tag Bettruhe hielt, fühlte sie sich viel besser.

Bei der Vorstellung im Juni 1899 giebt sie an, dass sie noch sehr oft beiderseits im Unterleib krampfartige Schmerzen hat, besonders bei körperlichen Anstrengungen. Besonders lebhafte Schmerzen empfindet sie bei der Cohabitation. Pat. ist immer "nicht ganz fest" gewesen, erklärt sich aber zur Zeit für leidlich wohl und arbeitsfähig.

Status: Uterus retrovertirt, flectirt, fixirt, nicht vergrössert. Beiderseits neben dem Uterus sind eirea hühnereigrosse, auf Druck wenig empindliche Tumoren zu füblen, àus welchen sich Tube und Ovarium nicht isolirt austasten lassen. Trotz häufiger Cohabitationen Sterilitä.t.

Fall 18. L., Luise, Kutschersfrau, 26 Jahre alt.

Poliklinische Behandlung October 1897.

Diaganose: Pyosalpinx dupl. gon.

Anamnese: Die Periode ist bis zuletzt regelmässig gewesen. Seit mehreren Wochen besteben zeitweise auftretende beiderseitige heftige Leib- und Kreuzschmerzen.

Status: Uterus nicht vergrössert, retrovertirt, fixirt. Beiderseits neben dem Uterus sind über hühnereigrosse Tumoren zu fühlen, aus denen sich Tube und Ovarien nicht isolirt austasten lassen und welche auf dem Beckenboden allseitig adhärent sind.

Bei der Vorstellung im Juni 1899 giebt Pat. an, dass ihre Periode regelmässig alle 4 Wochen mit geringem Blutverlust ist. Zeitweise hat Pat. noch während der Periode Kreuzschmerzen, Kopfsehmerzen und etwas Drängen nach unten. Bei grösseren körperlichen Anstrengungen treten manchmal rechterseits wie "rheumatische" Unterleibschmerzen ein. Pat. fühlt sich im Allgemeinen wohl und ist arbeitsfähig.

Status: Uterus retrovertirt, hinten fixirt. Rechte Tube und rechtes Ovarium fixirt, nicht vergrössert. Links neben dem Uterus ist ein etwa hühnereigrosser Tumor zu fühlen, aus welchem sich Tube und Ovarium nicht isolirt austasten lassen.

Fall 19. M., Lisbeth, Schriftsetzersehefrau, 23 Jahre alt.

Poliklinische Behandlung Juli 1897.

Diagnose: Pyosalpinx praecipue dextra. Sạlpingooophoritis sinistra. Endometritis cervicis gon., Pelviperitonitis subacuta.

Anamnese: Pat. hat 4 Frühgeburten mit normalen Wochenbetten durchgemacht mit Ausnahme des letzten im März 1897, wo Patientin 6 Wochen gelegen hat. Seit dieser Zeit bestehen Schmerzen im Unterleib und besonders in der rechten Seite.

Status: Rechts neben dem Uterus ist ein gänseeigrosser Tumor 
zu fühlen, welcher auf dem Beckenboden adhärent ist, und aus welchem sich Tube und Ovarinm nicht isolirt austasten lassen.

Die linke Tube ist deutlich verdickt zu fühlen; das linke Ovarium ist etwas vergxössert ebenfalls auf dem Beckenboden fixirt.

Bei der Vorstellung Juni 1899 giebt Pat. an, dass sie in der Zwischenzeit zweimal im Krankenhaus St. Jacob wegen Unterleibsbeschwerden gelegen hat, ohne wesentliche Besserung ihres Leidens. $\mathrm{Zu}$ Hause hat Pat. stets Sitzbäder und Scheidenspülungen gemacht. Die Periode ist unregelmässig, 5-8 Tage dauernd, sehr stark, meist mit krampfartigen Schmerzen verbunden. Pat. arbeitet zur Zeit als Anlegerin in einer Buchdruckerei, hat aber bei vielem Stehen und sonstigen geringen körperlichen Anstrengungen immer noch Lnterleibsschmerzen, besonders in der rechten Seite. Sie hat in der letzten Zeit noch Lues acquirirt und klagt seit der Zeit über. Mattigkeit und Appetitlosigkeit. Sic meint, dass keine Besserung eingetreten sei.

Fall 20. G., Ida, Puella publ., 23 Jahre alt.

Poliklinisch behandelt August 1897.

Diagnose: Pyosalpinx dupl. gon.

Anamnese: Beginn der Perjode mit 18 Jahren regelmässig alle 4. Wochen. Die letzte Periode März 1897, wie gewöhnlich.

Seit 10 Wochen keine Periode, dagegen ausserordentlich heftige Leibschmerzen.

Status: Uterus vergrössert, anteflectirt. Beiderseits neben dem Uterus sind Tumoren zu fühlen; aus denen sich Tube und Ovarien nicht isolirt austasten lassen, welche auf Druck ausserordentlich schmerzhaft sind. Im Urethralsecret zahlreiche Gonococcen nachweisbar.

Bei der Vorstellung im Juni 1899 giebt Pat. an, dass sie damals von der Poliklinik aus in das Krankenhaus St. Jacob geschickt ist, wo sie 12 Wochen lang mit Bettruhe und kalten Umschlägen und Scheideneinlagen behandelt wurde, ohne jede Besserung ihrer Beschwerden. Pat. machte dann im Bordell noch weiter täglich Scheidenspülungen. Die Periode blieb unregelmässig, manchmal längere Zeit aussetzend. Zu Weihnachten 1898 trat sie wiederum 4 Wochen lang ins Krankenhaus ein.

Sie klagt auch jetzt noch über Brennen beim Wasserlassen und fortwährende Schmerzen im Unterleib. Letztere treten besonders auch bei der Cohabitation auf, sodass diese in letzter Zeit überhaupt unmöglich wurde. Pat. fühlt sich, da sie, wie sie selbst zugiebt, sich körperlich gar nicht geschont habe und ausserdem häufige Cohabitationen ausgeführt, noch ebenso leidend, wie zu Anfang ihrer Erkrankung.

Status: Uterus antevertirt, nicht vergrösșert; ziemlich beweglich. Beide Tuben, am uterinen Ende verdickt, gehen beiderseits über in etwa hühnereigrosse, druckempfindliche Tumoren, aus denen sich die Ovarien nicht isolirt austasten lassen.

\section{Klinischer Verlauf nach Frühwochenbettsgonorrhoe.}

Fall 1. R., Ida, 29 Jahre alt. Erstgebärende. Geburt ${ }^{1}$ ) 8. 12. 1892. Temperatursteigerung im Wochenbett. Fieberabfall am 11. Tage. Einige

1. Anm.: Unter "Geburt" ist der Kürze halber im Folgenden die klinische Geburt gemeint, welcho von der Wochenbettsgonorrhoe gefolgt war. 
Zeit nach der Entbindung traten lebhafte Schmerzen im Unterleib auf, welche ärztliche Behandlung nothwendig machten. Diese war zunächst eine rein exspectative, dann aber wurde 2 Jahre nach der Geburt eine Laparotomie ausgeführt. Leider konnte man von dem behandelnden Arzt nicht erfahren, welcher Art der Eingriff gewesen war.

Pat. ist seit 1894 steril verheirathet. Periode ist unregelmässig; sonst ohne grössere Besehwerden. Zur Vorstellung 1899 konnte Pat. nicht bewogen werden, da sie ausswärts wohnte und die Reise scheute.

Fall 2. B., Antonie, 26 Jahre alt. Erstgebärende. Geburt 25. 12. 1892. Kein Fieber im Wochenbett. Nach $1 \frac{1 / 2}{2}$ Jahren wiederum Conception. Danach im nächsten Jahre noch zwei Kinder. Bei der Untersuchung waren die Tuben schlank und die Frau ohne Beschwerden.

Fall 3. L., Anna, 22 Jahre alt. Erstgebärende. Geburt 27. 12. 1892. Langauhaltendes Fieber im Wochenbett. Serös-eitriges Exsudat im Douglas, in weichem mikroskopisch und kulturell Gonococcen uachgewiesen wurden. Nach der Entlassung aus der Klinik hatte Pat. noch 1/2 Jahr lang Schmerzen im Unterleib. Die nächste Geburt erfolgte erst trotz häufiger Conceptionsmöglichkeit 3 Jahre später. Seit dieser Zeit bis 6. 1. 1899 drei Kinder. Die Untersuchung ergiebt Retroflexio uteri mobilis. Beide Tuben schlank, beide Ovarien klein, beweglich.

Fall 4. V., Ida, 27 Jahre alt. Heizersfrau. Viertgebärende. Letzte Geburt 2. 1. 1893. Nur einmalige Temperatursteigerung im Wochen'sett bis $38,1^{0}$. Entlassen am 10. Tage. Nachricht am 12. 3. 1899. Ist trotz häuffger Cohabitationen nicht wieder schwanger geworden. Beschwerden sind nicht vorhanden.

Fall 5. M., Anna, 22 Jahre alt, Dienstmagd. Erstgebärende. Geburt 7. 1. 1893. Einmalige Temperatursteigerung im Wochenbett bis $39,1^{\circ}$. Wieder vorgestellt am 17. 3. 1899. Hat keine Beschwerden gehabt. Ist im nächsten Jahre wieder schwanger geworden. Hat danach noch ausserdem 2 Entbindungen gehabt.

Fall 6. K., Fanny, 19 Jahre alt, Blumenbinderin. Erstgebärende. Geburt 8. 1. 1893. Leichtes Fieber im Frühwochenbett. Entlassung am 13. Tage. Vorstellung am 19. 4. 1899. Ist seit mehreren Jahren verheirathet. Geschlechtlicher Verkehr bestand seit der Entlassung: immer, aber keine Conception. Sie wurde im September 1898 wegen starken Ausflusses in der hiesigen Poliklinik behandelt. Die Diagnose daselbst lautet: Endometritis eum fluore. Adnexe ohne Besonderheiten. Erosio portionis.

Fall 7. B., Auguste, 19 Jahre alt, Sängerin. Erstgebärende. Geburt 9. 1. 1893. Temperaturstejgerungen im Frühwochenbett bis $39,0^{\circ}$. Fieberabfall am 10. Tage. Briefliche Mittheilung 23. 3. 1899. Nach der Entlassung noch mehrere Wochen erhebliche Schmerzen im Leibe. Auch in der Folgezeit plötzliche Schmerzattacken in der linken Seite. Geschlechtlicher Verkehr seit Mitte des Jahres 1893, häufig. Verheirathet seit Ende 1895. Trotz Wunsch nach Kindern keine Conception.

Fall 8. B., Louise, 26 Jahre alt, Plätterin. Zweitgebärende. Geburt 12. 1. 1893. Im Wochenbett lange Zeit krank gewesen, und zwar begann am 3. Tage lebhafter Schmer'z in Unterleib, welcher in der Folgezeit sich ansserordentlich steigerte mit Exacerbationen bei der Stuhlentleerung. Am 12. Tage wird in Narkose untersucht, weil die schmerzen immer stärker werden, und ein grosses Exsudat im Douglas 
festgestellt. Am 30. Tage des Wochenbetts nochmals Narkose. Der Uterus ist vorn anteponirt, die Tuben schlank, das Exsudat im Douglas über gänseeigross. Die Punction des Exsudats liefert eine eitrig-seröse Flüssigkeit, in welcher bei der Aussaat auf Agar mit Cystomflüssigkeit circa 60 Gonococcencolonien aufschiessen. Am 71. Tage des Wochenbetts wird sie ungeheilt zur poliklinischen Weiterhehandlung entlassen. Erst nach 7 Monaten ist sie so weit hergestellt, dass sie leichte Arbeiten verrichten kann; doch hat sie auch jetzt noch beständige Schmerzen. $11 / 2$ Jahr später sind die Schmerzen wesentlich geringer. $21 / 2$ Jahre später bei der Vorstellung giebt sic an, dass sie seit $3 / 4 \mathrm{Jahr}$ verheirathet sei, aber noch nicht concipirt hätte. Da in letzter Zeit wieder heftige Schmerzen aufgetreten sind, wird noch einmal narkotisirt. Der Uterus ist klein anteflectirt, beide Tuben ganz leicht verdickt. Das linke Ovarium in Verwachsungen eingebettet. Im Jahre 1897 ist sie zum ersten Male schwanger geworden und im Juni 1898 wiederum. Dann wieder im Jahre 1900, diesmal aber extrauterin. Wie ich einer Mitteilung von Herff aus Halle entnehme, ist sie daselbst wegen Tubenruptur linkerseits operirt worden.

Fall 9. H., Anna, 28 Jahre alt, Formersfrau. Geburt 19. 1. 1893. Leichte Temperatursteigerungen im Frühwochenbett. Entlassung am 10. Tage post partum. Nach der Entlassung noch 6 Wochen lang Schmerzen im Leibe und im Kreuz. $3 / 4$ Jahre nach der Entbindung schon wieder Conception und Entbindung im Jahre 1894 ohne jede Beschwerde. In der letzten Zeit nur selten geschlechtlicher Verkehr.

Am 9. 4. 1899: Uterus anteflectirt, klein, beweglich, beide Tuben schlank. Ovarien klein.

Fall 10. Z., Emma, 27 Jahre alt, Dienstmagd. Drittgebärende. Geburt 22. 1. 1893. Im Krühwochenbett Temperatursteigerungen bis 40,50, doch Abfall des Fiebers schon am 5. Tage. Bei der Entlassung normaler Genitaltastbefund. Ein Jahr später verheirathet. Bald nach der Verheirathung Conception und Geburt im Jahre 1895. Auch jetzt wieder 14 Tage lang im Wochenbett Fieber.

Die nächsten Entbindungen 1896 und 1898, jedes Mal mit Fieber im Wocbenbett verbunden. Zur Zeit Befinden gut. Untersuchung verweigert.

Fall 11. Sch., Clara, 18 Jahre alt, Fabrikarbeiterin. Erstgebärende. Geburt 25. 1. 1893. Temperatursteigerung im Frühwochenbett bis $40,2^{\circ}$. Fieberabfall allmälig am 22. Tage. Das Kind hat bei der Entlassung starke gonorrhoische Ophtbalmoblennorrhoe und Otitis media purulenta gonorrhoica. Nach der Entlassung hat Pat. noch 2 Jahre lang eitrigen Ausfluss. Nächste Entbindung schon im Februar 1894, die folgenden Entbindungen 1896 und 1898. Alle Kinder kamen im 8. Nonat faultodt.

Fall 12. St., Anna, $3 \breve{\partial}$ Jahre alt, Sattlersfrau. Fünftgebärende. Geburt 30. 1. 1893. Leichte Temperatursteigerung im Frühwochenbett. Entlassung am 10. Tage. Nach der Entbindung noch vorübergehende Schmerzen im Unterleibe. Geschlechtlicher Verkehr erst wieder seit Järz 1898. Bis 20. 4. 1899 ist keine Conception erfolgt. Periode regelmässig, keine Beschwerden. Untersuchung verweigert.

Fall 13. K., Emma, 22 Jahre alt. Erstgebärende. Geburt 31. 1. 1893. Im Frühwochenbett leichte Temperatursteigerung. Abfall des Fiebers am 3. Tage. 1/4 Jahr nach der Geburt geschlechtlicher Verkehr wieder aufgenommen. Nächste Entbindung im Jahre 1894. 
Wochenbett gut, ebenso die weiteren Entbindungen Juni 1897 und November 1898. Zur Zeit etwas Kreuzschmerzen.

Fall 14: S., Ida, 20 Jahre alt, Verkäuferin. Erstgebärende. Geburt 3. 2. 1893. Kein Fieber im Wochenbett. Noch 6 Wochen nach der Entlassung kränklich, und reichlich eitriger Ausfuss. Eine Conceptionsmöglichkeit ist erst seit $1 / 2$ Jabre wieder vorhanden; keine Gravidität. Die Untersuchung ergiebt: Uterus retrovertirt, lässt sich aufrichten. Beide Tuben am unterinen Ende leicht verdickt, gehen in taubeneigrosse Tumoren über, aus denen sich abdominelles Ende der Tuben und Ovarium nicht austasten lassen.

Fall 15. W., Minna, 20 Jahre alt, Arbeiterin. Erstgebärende. Geburt 17. 2. 1893. Temperatnrsteigerung in Frühwochenbett bis $39,5^{\circ}$. Abfall des Fiebers am 7. Tage. Entlassung. am 10. Tage. Nach der Entlassung noch lange Zeit Schmerzen in der rechten Unterleibsseite. Bis vor 2 Jahren immer ziemlich starker eitriger Ausfluss. Mit demselben Mann, von dem sie ihr erstes Kind hatte, häufige Cohabitationen ausgeführt bis vor 2 Jahren ohne jede Vorsichtsmassregel; trotzdem keine erneute Conception.

Am 18. 3. 1899: Schmerzen im Kreuz. Kopfschmerzen und etwas Ausfluss. Uterus klein, antevertirt, beweglich. Beide Tuben schlank. Beide Ovarien klein, beweglich.

Fall 16. W.. Minna, 24 Jahre alt, Dienstmagd. Zweitgebärende. Geburt 1. 3. 1893. Leichte Temperatursteigerung im Frühwochenbett. Entlassung am 10. Tage. Briefliche Mittheilung am 31. 7. 1899. Seit der Entbindmg gar keine Beschwerden. Emente Entbindung mit normal verlaufendem Wochenbett im Jahre 1898.

Fall 17. K., Pauline, 21 Jahre alt, Arbeiterin. Erstgebärende. Geburt 6. 3. 1893. Wieder vorgestellt am 12. 3. 1899. Zur Zeit verheirathet. Nach der Entlassung aus der Klinik 4 Wochen lang heftige Beschwerden. Der Arzt constatirte eine Eierstockentzündung. Cohabitationen in der Zwischenzeit sehr reichlich; doch keine Conception.

Unregelmässige Periode, und Schmerzen in der Gegend des rechten Unterleibs, ausserdem starke Kreuzschmerzen. Die Untersuchung ergiebt den Uterus retroflectirt. Adnexe beiderseits mit dem Uterus verwachsen, aber nicht vergrössert.

Fall 18. H., Marie, 28 J. alt, Näherin. Zweitgebärende. Geburt 11. 3. 1993. Im Wochen bett kein Fieber. Vorstellung am 20. 4. 1899. Nach der Entlassung immer noch Schmerzen im Unterleib. Ausfluss reichlich. Geschlechticher Verkehr 1 Jahr nach der Entbindung wieder aufgenommen, aber nicht häufig: keine Conception. Vom Arzt wegen Gebärmuttercatarrh und Blasenentzündung behandelt. Briefliche Mittheilung.

Fall 19. M., Anna. 22 J. alt, Fabrikarbeiterin. Zweitgebärende. Geburt 14. 3. 1893. Leichte Temperatursteigerung im Wochenbett. Nach der Entlassung zunächst Wohlbefinden. Im September 1894 Schmerzen in der rechten Seite des Unterleibs, besonders beim Stuhlgang. Austluss immer ziemlich reichlich. Geschlechtlicher Verkehr $1 / 2$ J. nach der Entbindung. Conception bis 17. 4. 1899 nicht erfolgt. Status vom 17. 4. 1899: Uterus antevertirt, klein, beweglich. Linke Tube schlank. Ovarium klein, beweglich. Rechte Tube vielleicht etwas verdickt, Ovarium aber klein, beweglich.

Fall 20. S., Therese, $28 \mathrm{~J}$. alt. Zimmermannsfrau. Viertgebärende. Geburt 15. 3. 1893. Im Wochenbett kein Fieber. Wieder vorgestellt 
am 6. 1. 1899. Nach der Entlassung keine weiteren Beschwerden gehabt, auch keinen Ausfuss. Nächste Entbindung 31. 12. 1895. Dawach 14 Wochen lang Fieber. Die nächste Entbindung 19. 3. 1897. Acht Wochen lang Fieber. Letzte Entbindung vor 19 Wochen. 7 Wochen lang Fieber. Geburten stets normal. Die innere Untersuchung ergiebt Uterus anteflectirt, frei beweglich. Beide Tuben schlank, Ovarium klein; frei beweglich.

Fall 21. G., Anna, 21 J. alt. Arbeiterin. Erstgebärende. Geburt 4. 3. 1893. Leichte Temperatursteigerungen im Wochenbett. Entlassung am 10. Tage. Vorstellung am 25. 3. 1899. Seit der Entbindung immer geschlechtlicher Verkehr. Seit $1 \mathrm{~J}$. verheirathet. Nach der Entlassung noch Schmerzen im Leibe, die sich 10 Wochen nach der Entbindung so steigerten, dass Pat. das Krankenhaus aufsuchen musste. Hier wurde sie 4 Wochen lang mit Umschlägen auf den Leib behandelt, worauf Linderung eintrat. Nach $2 \mathrm{~J}$. wegen eneuter Schmerzen Wiederaufnahme in eine Klinik und dort 4 Wochen lang in gleicher Weise behandelt. Die Schmerzen kehrten jedoch immer wieder. Der Mamn ist öfter wegen Harnröhrenleiden in Behandlung gewesen. Conception ist nicht wieder eingetreten.

Uterus klein, anteflectirt, frei beweglich. Starke Erosion an der Portio. Beide Tuben schlank, beide Ovarien klein, beweglich.

Fall 22. L., Anna, 25 J. alt. Dienstmagd. Erstgebärende. Geburt 25. 4. 1893. Leichte Temperatursteigerung im Frübwochenbett. Entlassung am 10. Tage.

Vorstellung 10. 3. 1899. Seit der Entlassung immer Beschwerden, und zwar Ziehen im Leibe, Schmerzen im Rücken mit starkem weissem Ausfluss verbunden. Periode unregelmässig; schmerzhaft. Seit Januar 1895 verheirathet. Cohabitationen aber schon früher. Fine Conception erfolgte im October 1895; Abort von 6 Wochen. Danach wiederum sehr heftige Beschwerden. Seit der Zeit nie wieder concipirt. Untersuchung ergiebt; Uterus klein, breit, hinten fixirt. Tuben und Ovarien nicht vergrössert.

Fall 23. B., Anna, 19 J. alt. Falzerin. Erstgebärende. Geburt 4. 6. 1893, Temperatursteigerung im Wochenbett bis über $39^{\circ}$. Fieberabfall erst am 20. Tage.

Vorstellung am 30. 4. 1899. Nach der Entlassung noch einige Tage Schmerzen im Unterleib. Geschlechtlicher Verkehr 3 Monate nach der Geburt wieder aufgenommen, aber nur selten. Nächste Entbindung Januar 1896 in der Klinik ohne Fieber. Weitere Entbindung August 1897.

Innere. Untersuching verweigert.

Fall 24. K., Auguste, 21 J. alt, Dienstmagd. Zweitgebärende. Geburt 30. 7. 1893. Kein Fieber im Wochenbett. Nach der Entbindung anfänglich Wohlbefinden. Im December 1893 treten lebhafte Schmerzen im Unterleib mit unregelmässigen Blutungen ein. Am 18. Januar 1894 wurde Pat. wegen gonorrhoischer Pyosalpinx operirt. Es war rechts am Uterus ein gänseeigrosses und links ein faustgrosses Convolut von Tumoren vor der Operation zu constatiren. Die Operation bestand in Salpingooophorectomia bilateralis. Anatomische Diagnose: Gonorrh. Pyosalpynx. Nach der Operation noch $2 \mathrm{~J}$. Iang geringe Beschwerden. Auch jetzt noch (10.4. 1899) geringe Spannung im Leib, ausserdem Kopfschmerzen, fliegende Hitze und Schmerzen in der linken Seite des 
Unterleibs. Der Uterus ist sehr klein, anteflectirt, beweglich. Adnexe nicht zu fühlen.

Fall 25. K., Anna, 25 J. alt. Arbeiterin. Erstgebärende. Geburt 26. 9. 1893. Hohes Fieber im Frühwochenbett. Abfall am 14. Tage. Entlassung am 16. Tage.

Vorstellung 31. 7. 1899. Nach der Entlassung keine Beschwerden mehr. Geschlechtlicher Verkehr seit 9 Jahren. Verheirathet seit November 1897. Erste weitere Entbindung März 1898; ohne Fieber. Die nächste Entbindung Februar 1899.

Die Untersuchung ergiebt: Uterus klein, anteflectirt, beweglich. Adnexe olne Besonderheiten.

Fall 26. K., Margarete, 19 J. alt. Kellnerin. Erstgebärende. Geburt 28. 9. 1893. Hohe Temperatursteigerung im Frühwochenbett. Fieberabfall am 18. Tage. Rectalgonorrhoe. Entlassung 27 Tage nach der Entbindung. Briefliche Mittheilung 15. 3. 1899, aus welcher nur entnommen werden kam, dass sie z. Z. schwanger ist.

Fall 27. R., Katharine, 23. J. alt. Arbeiterin. Zweitgebärende. Geburt 6. 12. 1893. Hohe Temperatursteigerung im Frühwochenbett. Fieberabfall an 11. Tage. Entlassung am 13. Tage.

Vorstellung am 29. 3. 1899.

Die erste Zeit nach der Entlassung keine Beschwerden; November 1895 angeblich Blinddarmentzündung. Zweimal wegen heftiger Schmerzen im Unterleib im Krankenhause behandelt. Geschlechtlicher Verkehr bestand immer, allerdings selten; im allgemeinen nicht mehr als zweimal im Monat. Seit Februar 1897 verheirathet, doch wurde der Ehemann bald danach im Zuchthaus internirt.

Uterus ist nicht vergrössert, retoflectirt, hinten brejt fixirt. Links vom Uterus ist ein gänseeigrosser Tumor zu fühlen, welcher mit dem Beckenboden allseitig verwachsen ist und dem Uterus direkt anliegt; aus ihm lassen sich Tube und Ovarium nicht isolirt austasten. Die rechte Tube ist am uterinen Fnde noch eine kurze Strecke hin verdickt zu füblen, geht aber dann über auf das Ovarium, welches auf dem Beckenboden ebenfalls fest verwachsen ist.

Fall 28. P.. Anna, 33 J. alt. Dienstmädehen. Erstgebärende. Geburt 1. 7. 1893. Kein Fieber im Wochenbett. Entlassung am 10. Tage. Nach der Entlassung keine Beschwerden. Nächste Entbindung April 1896.

Frühgeburt wegen Syphilis im Mai 1898.

Fall 29. P., Selma, 18. J. alt. Schneiderstochter. Erstgebärende. Gehurt 20. 10. 1893. Kein Fieber. Entlassung am 10. Tage. Geschlechtlicher Verkehr bald nach der Entbindung. Nächste Entbindung im Mai 1895. Bis zum 29. 3. 1899 noch zwei weitere Entbindungen. Immer beschwerdefrei.

Fall 30. F., Marie, 21. J. alt, Kellnerin. Erstgebärende. Geburt 4. 1. 1894. Leichte Temperatursteigerung im Frühwochenbett. Entlassung am 10. Tage.

Vorstellung am 7. 3. 1899. Periode nach der Entbindung unregelmässig und schmerzhaft. Cohabitationen sind sofort nach der Entbindung wieder aufgenommen, häufig, aber keine Conception. Am 24. 4. 1897 wird wegen hauptsächlich rechtsseitiger Pyosalpinx die Salpingooophorectomia dextra ansgeführt. Bei der Untersuchung am 7. 3.1899 ist der Uterus vorn; die linken Adnexe sind wegen leichter Schmerzhaftigkeit nicht sicher zu tasten. 
Fall 31. Ch., Adelaide, $24 \mathrm{~J}$. alt. Dienstmädehen. Erstgebärende. Geburt 8. 1. 1894. Kein. Fieber. Entlassung am 10. Tage. Cohabitationen gleich nach der Entbindung wieder aufgenommen; sehr reichlich. Erste weitere Entbindung 1897. Seit der Zeit nicht wieder geschwängert trotz häufiger Cohabitationen. Keine Beschwenden. Uterns vorn, klein. Adnexe ohne Besonderheiten.

Fall 32. R., Auguste, 26 J. alt. Arbeiterin. Zweitgebärende. Geburt.19. 1. 1894. Leichte Temperatursteigerung im Frühwochenbett. Entlassung am 10. Tage. Nach 3 Wochen wiederum in die Klinik aufgenommen and zwar auf die gypäkologische Abtheilung wegen heftiger Schmerzen in Unterleib. Uterus damals vergrössert, anteflectirt: Beide Tuben leicht verdickt, aber beweglich.

Nach 14tägiger Behandlung auf der gynäkologischen Station sind die Beschwerden wesentlich gebessert. Geschlechtlicher Verkehr erst seit Anfang 1896 wieder aufgenommen. Nächste Entbindung Januar 1897. Anch jetzt im Wochenbett Fieber und Schmerzen, welche nach 6 Wochen sich besserten. Nächste Entbindung Januar1 898.

Uterus ist klein, anteflectirt, beweg]ich. Beide Tuben schlank. Ovarien klein.

Fall 33. W., Emma, 25 J. alt. Arbeiterin. Zweitgebärende. Geburt am 2. 2. 1894. Leichte Temperatursteigerungen im Frühwochenbett. Entlassung am 14. Tage. Einige Wochen nach der Entlassung traten wieder heftige Schmerzen im Unterleib ein, sodass Pat. ganz gebiickt gehen musste. In Wien in der Klinik Chrobak's wegen Eitercatarrhs mit Sitzbädern und Ausspülungen behandelt, worauf Beschwerden bis zum Mai 1891 rollständig zurüekgingen. Ausfluss besteht auch jetz noch immer. Pat. ist seit Juli $189 \bar{z}$ verheirathet; seitdem geschlechtlicher Verkehr, aber erst jetzt, 23. 3. 1899, ist Pat. zum ersten Mal schwanger im 9. Monat.

Fall 34. K., Bertha, 25. J. alt. Arbeiterin. Erstgebärende. Geburt 14. 2. 1894. Im Wochenbett kein Fieber. Entlassung am 10. Tage. $1 / 4$ J. nach der Entbindung geschlechtlicher Verkehr wieder häufig. Erste weitere Geburt Mai 1898, ohne Fieber im Wochenbert. Zur Zeit (10. 7. 1899) keine Beschwerden.

Uterus klein, antervertirt, beweglich. Adnexe ohne Besonderheiten.

Fall 35. S., Anna, 25 J. alt. Arbeiterin. Erstgebärende. Geburt 15. 2. 1894. Leichte Temperatursteigerung im Frühwochenbett. Entlassung am 10. Tage. Vorstellung am 4. 3. 1899. Seit der Entlassung keine Beschwerden. Conceptionsmöglichkeit reichlich vorhanden. Trotzdem erst Anfang 1899 die erste Entbindung.

Uterus klein, frei beweglich. Adnexe ohne Besonderheiten.

Fall 36. F., Bertha, 29 J. alt. Arbeiterin. Viertgebärende. Geburt 26. 2. 1894. Kein Fieber im Wochenbett. Es konnte nur in Erfahrung gebracht werden, dass sie Anfang 1899 eine Entbindung am Ende der Zeit durchgemacht hatte. 